Supplemental materials of

\title{
Active cigarette smoking is associated with an exacerbation of genetic susceptibility to diabetes
}

Wan-Yu Lin ${ }^{1,2 *}$, Yu-Li Liu ${ }^{3}$, Albert C. Yang ${ }^{4,5}$, Shih-Jen Tsai ${ }^{5,6,7}$, Po-Hsiu Kuo ${ }^{1,2 *}$

${ }^{1}$ Institute of Epidemiology and Preventive Medicine, College of Public Health, National Taiwan University, Taipei, Taiwan

${ }^{2}$ Department of Public Health, College of Public Health, National Taiwan University, Taipei, Taiwan

${ }^{3}$ Center for Neuropsychiatric Research, National Health Research Institutes, Miaoli, Taiwan

${ }^{4}$ Division of Interdisciplinary Medicine and Biotechnology, Beth Israel Deaconess Medical

Center/Harvard Medical School, Boston, MA, USA

${ }^{5}$ Institute of Brain Science, National Yang-Ming University, Taipei, Taiwan

${ }^{6}$ Division of Psychiatry, National Yang-Ming University, Taipei, Taiwan

${ }^{7}$ Department of Psychiatry, Taipei Veterans General Hospital, Taipei, Taiwan

Short title: Smoking and genetic risk of diabetes

* Corresponding authors: Po-Hsiu Kuo, Ph.D. and Wan-Yu Lin, Ph.D.

Po-Hsiu Kuo, Ph.D. (http://orcid.org/0000-0003-0365-3587)

Room 521, No. 17, Xu-Zhou Road, Taipei 100, Taiwan

Phone: +886-2-33668015; Fax:+886-2-23511955; E-mail: phkuo@ntu.edu.tw

Wan-Yu Lin, Ph.D. (http://orcid.org/0000-0002-3385-4702)

Room 501, No. 17, Xu-Zhou Road, Taipei 100, Taiwan

Phone/Fax: +886-2-33668106; E-mail: linwy@,ntu.edu.tw 


\begin{tabular}{|c|c|c|c|c|c|c|}
\hline $\begin{array}{c}\text { (Discovery cohort / Replication cohort) } \\
\text { (D/R) }\end{array}$ & \multicolumn{2}{|c|}{ Fasting glucose (mg/dL) } & \multicolumn{2}{|c|}{ HbA1c (\%) } & \multicolumn{2}{|c|}{ Diabetes (dichotomous trait) } \\
\hline $\begin{array}{l}\text { Explanatory variables in regression } \\
\text { model (1) }\end{array}$ & Percent change (\%) & $p$-value & $\begin{array}{c}\text { Percent } \\
\text { change (\%) }\end{array}$ & $p$-value & Odds ratio & $p$-value \\
\hline $\begin{array}{c}\text { Sex } \\
\text { (1: female vs. 0: male) }\end{array}$ & $-3.71^{2}(-3.33)$ & $\begin{array}{c}4.5 \mathrm{E}-77 \\
(2.8 \mathrm{E}-124)\end{array}$ & $-0.61(-0.81)$ & $\begin{array}{c}3.4 \mathrm{E}-5 \\
(5.9 \mathrm{E}-15)\end{array}$ & $0.73(0.71)$ & $\begin{array}{l}9.7 \mathrm{E}-10 \\
(1.3 \mathrm{E}-24)\end{array}$ \\
\hline $\begin{array}{l}\text { Age } \\
\text { (in years, continuous variable) }\end{array}$ & $0.34(0.36)$ & $4.8 \mathrm{E}-272(0)$ & $0.28(0.29)$ & $0(0)$ & $1.08(1.08)$ & $3.5 \mathrm{E}-177(0)$ \\
\hline $\begin{array}{c}\text { Body mass index } \\
\text { (in } \mathrm{kg} / \mathrm{m}^{2} \text {, continuous variable) }\end{array}$ & $0.87(0.85)$ & $1.3 \mathrm{E}-239(0)$ & $0.70(0.73)$ & $\begin{array}{c}3.1 \mathrm{E}-297 \\
(0)\end{array}$ & $1.18(1.18)$ & 1.7E-155 (0) \\
\hline $\begin{array}{l}\text { The number of pack-years } \\
\text { (mean } \pm \text { sd, D: } 19.6 \pm 17.0 ; \text { R: } 19.5 \pm 17.6 \text { ) }\end{array}$ & $0.06(0.05)$ & $2.3 \mathrm{E}-7(8.8 \mathrm{E}-9)$ & $0.06(0.06)$ & $\begin{array}{l}4.0 \mathrm{E}-12 \\
(5.8 \mathrm{E}-22)\end{array}$ & $1.01(1.01)$ & $5.9 \mathrm{E}-9(6.5 \mathrm{E}-12)$ \\
\hline $\begin{array}{l}\text { Drinking status } \\
\text { (1: yes vs. 0: no) }\end{array}$ & $1.18(1.48)$ & $1.6 \mathrm{E}-3(1.2 \mathrm{E}-7)$ & $-1.07(-1.32)$ & $\begin{array}{c}7.0 \mathrm{E}-5 \\
(5.1 \mathrm{E}-11)\end{array}$ & $0.98(0.88)$ & $0.83(0.05)$ \\
\hline $\begin{array}{l}\text { Regular exercise } \\
\text { (1: yes vs. 0: no) }\end{array}$ & $-0.62(-0.87)$ & $\begin{array}{c}1.8 \mathrm{E}-3 \\
(2.8 \mathrm{E}-11)\end{array}$ & $-0.88(-0.95)$ & $\begin{array}{c}1.1 \mathrm{E}-9 \\
(1.3 \mathrm{E}-23)\end{array}$ & $0.89(0.87)$ & $0.02(1.4 \mathrm{E}-5)$ \\
\hline $\begin{array}{l}\text { Educational attainment } \\
\text { (a value ranging from } 1 \text { to } 7 \text { ) }\end{array}$ & $-0.57(-0.51)$ & $\begin{array}{c}6.6 \mathrm{E}-8 \\
(7.0 \mathrm{E}-14)\end{array}$ & $-0.48(-0.19)$ & $\begin{array}{l}3.9 \mathrm{E}-10 \\
(1.0 \mathrm{E}-4)\end{array}$ & $0.92(0.89)$ & $3.2 \mathrm{E}-4$ (2.7E-15) \\
\hline R-square & $13.8 \%$ & .1\%) & $14.2 \%$ & $3.9 \%)$ & 13.8 & $13.3 \%)$ \\
\hline
\end{tabular}

Supplemental Table S1 Results of regression model (1) when the smoking measurement is "the number of pack-years" (prior to GRS analysis) 


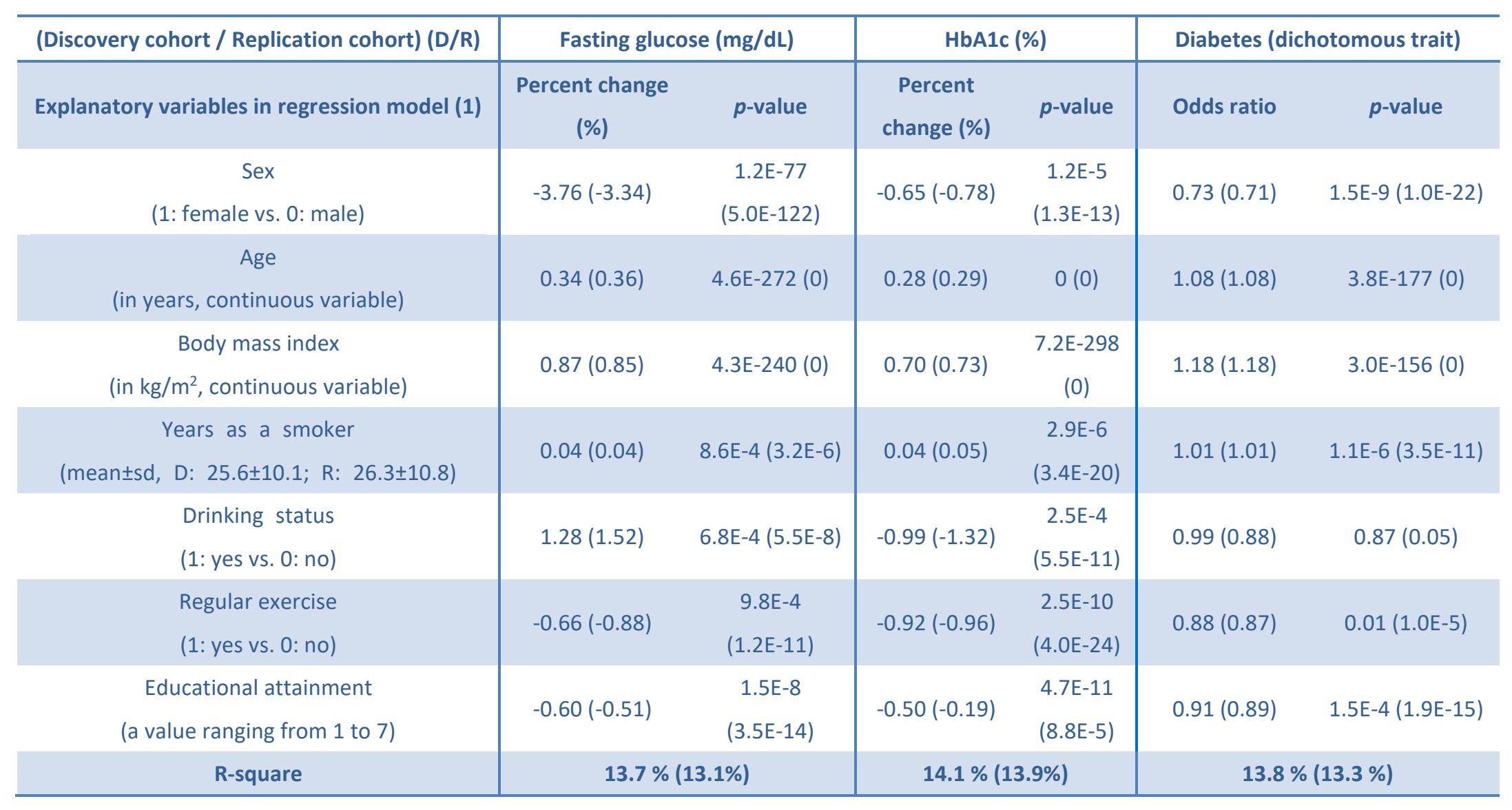

Supplemental Table S2 Results of regression model (1) when the smoking measurement is "the number of years as a smoker" (prior to GRS analysis) 


\begin{tabular}{|c|c|c|c|c|c|c|}
\hline \multirow{2}{*}{$\begin{array}{l}\text { (Discovery cohort / Replication cohort) (D/R) } \\
\text { Explanatory variables in regression model (1) }\end{array}$} & \multicolumn{2}{|c|}{ Fasting glucose (mg/dL) } & \multicolumn{2}{|c|}{ HbA1c (\%) } & \multicolumn{2}{|c|}{ Diabetes (dichotomous trait) } \\
\hline & $\begin{array}{l}\text { Percent change } \\
\text { (\%) }\end{array}$ & $p$-value & $\begin{array}{c}\text { Percent } \\
\text { change (\%) }\end{array}$ & $p$-value & Odds ratio & $p$-value \\
\hline $\begin{array}{c}\text { Sex } \\
\text { (1: female vs. 0: male) }\end{array}$ & $-3.70(-3.35)$ & $\begin{array}{c}1.5 \mathrm{E}-76 \\
(4.1 \mathrm{E}-125)\end{array}$ & $-0.61(-0.82)$ & $\begin{array}{c}3.6 \mathrm{E}-5 \\
(2.6 \mathrm{E}-15)\end{array}$ & $0.73(0.71)$ & $\begin{array}{c}1.5 \mathrm{E}-9 \\
(9.0 \mathrm{E}-25)\end{array}$ \\
\hline $\begin{array}{l}\text { Age } \\
\text { (in years, continuous variable) }\end{array}$ & $0.35(0.36)$ & $\begin{array}{c}1.7 E-276 \\
(0)\end{array}$ & $0.29(0.29)$ & $0(0)$ & $1.08(1.08)$ & $3.1 \mathrm{E}-181(0)$ \\
\hline $\begin{array}{c}\text { Body mass index } \\
\text { (in } \mathrm{kg} / \mathrm{m}^{2} \text {, continuous variable) }\end{array}$ & $0.87(0.85)$ & $\begin{array}{c}7.2 \mathrm{E}-239 \\
(0)\end{array}$ & $0.70(0.73)$ & $\begin{array}{c}3.5 \mathrm{E}-296 \\
(0)\end{array}$ & $1.18(1.18)$ & $1.3 \mathrm{E}-154(0)$ \\
\hline $\begin{array}{c}\text { Packs smoked per day } \\
\text { (mean } \pm \text { sd, D: } 0.72 \pm 0.51 ; R: 0.69 \pm 0.52 \text { ) }\end{array}$ & $1.70(1.33)$ & $\begin{array}{c}4.9 \mathrm{E}-7 \\
(3.6 \mathrm{E}-7)\end{array}$ & $1.63(1.70)$ & $\begin{array}{c}3.4 \mathrm{E}-11 \\
(7.0 \mathrm{E}-19)\end{array}$ & $1.51(1.41)$ & $\begin{array}{c}1.1 \mathrm{E}-9 \\
(2.3 \mathrm{E}-11)\end{array}$ \\
\hline $\begin{array}{l}\text { Drinking status } \\
\text { (1: yes vs. 0: no) }\end{array}$ & $1.17(1.49)$ & $\begin{array}{l}1.9 \mathrm{E}-3 \\
(9.3 \mathrm{E}-8)\end{array}$ & $-1.08(-1.31)$ & $\begin{array}{c}6.0 \mathrm{E}-5 \\
(6.9 \mathrm{E}-11)\end{array}$ & $0.97(0.88)$ & $0.76(0.05)$ \\
\hline $\begin{array}{l}\text { Regular exercise } \\
\text { (1: yes vs. 0: no) }\end{array}$ & $-0.63(-0.88)$ & $\begin{array}{c}1.6 \mathrm{E}-3 \\
(1.6 \mathrm{E}-11)\end{array}$ & $-0.89(-0.96)$ & $\begin{array}{l}7.1 \mathrm{E}-10 \\
(3.9 \mathrm{E}-24)\end{array}$ & $0.89(0.87)$ & $0.02(1.2 \mathrm{E}-5)$ \\
\hline $\begin{array}{l}\text { Educational attainment } \\
\text { (a value ranging from } 1 \text { to } 7 \text { ) }\end{array}$ & $-0.56(-0.50)$ & $\begin{array}{c}1.0 \mathrm{E}-7 \\
(1.1 \mathrm{E}-13)\end{array}$ & $-0.47(-0.19)$ & $\begin{array}{l}7.0 \mathrm{E}-10 \\
(1.6 \mathrm{E}-4)\end{array}$ & $0.92(0.89)$ & $\begin{array}{c}4.8 \mathrm{E}-4 \\
(4.6 \mathrm{E}-15)\end{array}$ \\
\hline R-square & $13.8 \%$ (1) & & $14.2 \%$ & $3.9 \%)$ & $13.8 \%$ & $3.3 \%)$ \\
\hline
\end{tabular}

Supplemental Table S3 Results of regression model (1) when the smoking measurement is "packs smoked per day" (prior to GRS analysis) 


\begin{tabular}{|c|c|c|c|c|c|c|}
\hline \multirow{2}{*}{$\begin{array}{l}\text { (Discovery cohort / Replication cohort) (D/R) } \\
\text { Explanatory variables in regression model (1) }\end{array}$} & \multicolumn{2}{|c|}{ Fasting glucose (mg/dL) } & \multicolumn{2}{|c|}{ HbA1c (\%) } & \multicolumn{2}{|c|}{ Diabetes (dichotomous trait) } \\
\hline & $\begin{array}{c}\text { Percent } \\
\text { change (\%) }\end{array}$ & $p$-value & $\begin{array}{c}\text { Percent } \\
\text { change (\%) }\end{array}$ & $p$-value & Odds ratio & $p$-value \\
\hline $\begin{array}{c}\text { Sex } \\
\text { (1: female vs. 0: male) }\end{array}$ & $-3.92(-3.51)$ & $\begin{array}{c}1.2 \mathrm{E}-89 \\
(4.6 \mathrm{E}-145)\end{array}$ & $\begin{array}{l}-0.82 \\
(-1.03)\end{array}$ & $\begin{array}{c}1.1 \mathrm{E}-8 \\
(1.4 \mathrm{E}-24)\end{array}$ & $0.68(0.67)$ & 9.7E-14 (1.8E-33) \\
\hline $\begin{array}{c}\text { Age } \\
\text { (in years, continuous variable) }\end{array}$ & $0.34(0.36)$ & $\begin{array}{c}1.2 \mathrm{E}-272 \\
(0)\end{array}$ & $0.28(0.29)$ & $0(0)$ & $1.08(1.08)$ & $1.6 \mathrm{E}-177(0)$ \\
\hline $\begin{array}{c}\text { Body mass index } \\
\text { (in } \mathrm{kg} / \mathrm{m}^{2} \text {, continuous variable) }\end{array}$ & $0.87(0.85)$ & $\begin{array}{c}5.3 \mathrm{E}-240 \\
(0)\end{array}$ & $0.70(0.73)$ & $\begin{array}{l}\text { 8.7E-298 } \\
\text { (0) }\end{array}$ & $1.18(1.18)$ & $4.2 \mathrm{E}-156(0)$ \\
\hline $\begin{array}{l}\text { Hours as a passive smoker per week } \\
\text { (mean } \pm \text { sd, D: } 5.6 \pm 11.0 \text {; R: } 5.4 \pm 10.6 \text { ) }\end{array}$ & $0.03(0.04)$ & $0.18(0.02)$ & $0.01(0.03)$ & $\begin{array}{c}0.52 \\
(0.03)\end{array}$ & $1.01(1.01)$ & $0.04(0.01)$ \\
\hline $\begin{array}{l}\text { Drinking status } \\
\text { (1: yes vs. 0: no) }\end{array}$ & $1.47(1.71)$ & $\begin{array}{c}8.0 \mathrm{E}-5 \\
(5.0 \mathrm{E}-10)\end{array}$ & $\begin{array}{l}-0.78 \\
(-1.01)\end{array}$ & $\begin{array}{l}3.6 \mathrm{E}-3 \\
(3.1 \mathrm{E}-7)\end{array}$ & $1.04(0.94)$ & $0.67(0.29)$ \\
\hline $\begin{array}{l}\text { Regular exercise } \\
\text { (1: yes vs. 0: no) }\end{array}$ & $-0.70(-0.92)$ & $\begin{array}{c}4.0 \mathrm{E}-4 \\
(1.2 \mathrm{E}-12)\end{array}$ & $\begin{array}{l}-0.97 \\
(-1.02)\end{array}$ & $\begin{array}{c}2.3 \mathrm{E}-11 \\
(3.9 \mathrm{E}-27)\end{array}$ & $0.87(0.85)$ & $0.005(9.3 E-7)$ \\
\hline $\begin{array}{c}\text { Educational attainment } \\
\text { (a value ranging from } 1 \text { to } 7 \text { ) }\end{array}$ & $-0.63(-0.53)$ & $\begin{array}{c}2.5 \mathrm{E}-9 \\
(2.8 \mathrm{E}-15)\end{array}$ & $\begin{array}{l}-0.54 \\
(-0.23)\end{array}$ & $\begin{array}{l}1.7 \mathrm{E}-12 \\
(3.6 \mathrm{E}-6)\end{array}$ & $0.91(0.88)$ & $3.7 \mathrm{E}-5(8.0 \mathrm{E}-17)$ \\
\hline R-square & \multicolumn{2}{|c|}{$13.7 \%$ (13.1 \%) } & \multicolumn{2}{|c|}{$14.0 \%(13.8 \%)$} & \multicolumn{2}{|c|}{$13.6 \%(13.2 \%)$} \\
\hline
\end{tabular}

Supplemental Table S4 Results of regression model (1) when the smoking measurement is "hours as a passive smoker per week" (prior to GRS analysis) 


\begin{tabular}{|c|c|c|c|c|c|c|c|c|c|c|c|}
\hline Chr. & SNP / SNV & Base pair & $\begin{array}{l}\text { Effect } \\
\text { allele }\end{array}$ & $\begin{array}{l}\text { Other } \\
\text { allele }\end{array}$ & Mapped gene & MAF & $\begin{array}{l}P_{I N T} \text { with } \\
\text { "active smoking } \\
\text { status" }\end{array}$ & $\begin{array}{l}P_{I N T} \text { with "the } \\
\text { number of } \\
\text { pack-years" }\end{array}$ & $\begin{array}{l}P_{I N T} \text { with } \\
\text { "years as a } \\
\text { smoker" }\end{array}$ & $\begin{array}{l}P_{I N T} \text { with "packs } \\
\text { smoked per day" }\end{array}$ & $\begin{array}{l}P_{I N T} \text { with } \\
\text { "hours as a } \\
\text { passive smoker } \\
\text { per week" }\end{array}$ \\
\hline 2 & rs340515 & 45188370 & G & $\mathrm{T}$ & ------ & 0.439523 & 0.421814 & 0.369612 & 0.248795 & 0.508034 & 0.237799 \\
\hline 2 & rs34177044 & 169754485 & $A$ & G & ------ & 0.403827 & 0.390463 & 0.011082 & 0.022235 & 0.117793 & 0.472982 \\
\hline 2 & rs2232326 & 169764491 & C & $\mathrm{T}$ & G6PC2 & 0.041328 & 0.202882 & 0.203321 & 0.221588 & 0.3382 & 0.805891 \\
\hline 2 & rs60415045 & 169771124 & C & $A$ & ------ & 0.286759 & 0.18333 & 0.019855 & 0.032892 & 0.142232 & 0.241038 \\
\hline 6 & rs9356744 & 20685486 & C & $\mathrm{T}$ & CDKAL1 & 0.348777 & 0.199681 & 0.20419 & 0.191177 & 0.083728 & 0.983701 \\
\hline 7 & rs730497* & 44223721 & A & G & GCK & 0.196822 & 0.21977 & 0.225264 & 0.512717 & 0.051395 & 0.553832 \\
\hline 7 & rs13229610 & 44258964 & $\mathrm{~T}$ & G & CAMK2B & 0.459106 & 0.335828 & 0.049154 & 0.029717 & 0.274366 & 0.644279 \\
\hline 7 & rs2233580** & 127253550 & $\mathrm{~T}$ & C & PAX4 & 0.106884 & 0.02893 & 0.077768 & 0.046995 & 0.097878 & 0.783261 \\
\hline 7 & rs61342118* & 127761917 & A & C & Near PAX4 & 0.086016 & 0.026518 & 0.088693 & 0.04746 & 0.037248 & 0.549475 \\
\hline 8 & rs75199970 & 2546494 & $\mathrm{~T}$ & $A$ & LOC101927815 & 0.000230 & 0.040606 & 0.03539 & 0.041408 & 0.035423 & 0.67093 \\
\hline 8 & rs35859536* & 118191475 & $\mathrm{~T}$ & C & Near SLC30A8 & 0.462279 & 0.672247 & 0.068205 & 0.105456 & 0.318498 & 0.109532 \\
\hline 9 & rs10811661* & 22134094 & C & $\mathrm{T}$ & ------ & 0.416026 & 0.430898 & 0.124122 & 0.424267 & 0.347333 & 0.361638 \\
\hline 10 & rs2399794 & 12248800 & G & A & CDC123 & 0.473194 & 0.028171 & 0.000861 & 0.038414 & $9.68 \mathrm{E}-05$ & 0.98143 \\
\hline 11 & rs163177 & 2838413 & C & $\mathrm{T}$ & KCNQ1 & 0.465963 & 0.366676 & 0.101701 & 0.100647 & 0.126057 & 0.316126 \\
\hline 11 & rs60808706 & 2857233 & $A$ & G & KCNQ1 & 0.358092 & 0.409672 & 0.131914 & 0.49631 & 0.143097 & 0.147599 \\
\hline 11 & rs10830963 & 92708710 & G & C & MTNR1B & 0.431628 & 0.900442 & 0.440297 & 0.274092 & 0.899981 & 0.535962 \\
\hline
\end{tabular}

Supplemental Table S5 16 FG-associated SNPs discovered by TWB1 $\left(P_{S N P}<6.25 \times 10^{-9}\right.$ in model 2)

** rs2233580 was also HbA1c-associated $\left(P_{S N P}<6.25 \times 10^{-9}\right.$ in model 2) and diabetes-associated $\left(P_{S N P}<6.25 \times 10^{-9}\right.$ in model 2$)$.

* rs61342118 was also diabetes-associated $\left(P_{S N P}<6.25 \times 10^{-9}\right.$ in model 2).

* rs730497, rs35859536, and rs10811661 were also HbA1c-associated $\left(P_{S N P}<6.25 \times 10^{-9}\right.$ in model 2). 
$P_{I N T}$ is the $p$-value of testing $H_{0}: \beta_{I N T}=0$ vs. $H_{1}: \beta_{I N T} \neq 0$ from the following model:

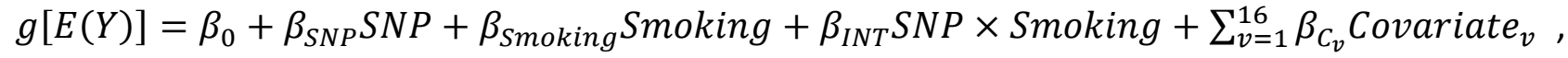

where $Y$ is natural log transformed fasting glucose with an identity link $g[\cdot]$, and Smoking is one of the five smoking measurements. 


\begin{tabular}{|c|c|c|c|c|c|c|c|c|c|c|c|}
\hline Chr. & SNP / SNV & Base pair & $\begin{array}{l}\text { Effect } \\
\text { allele }\end{array}$ & $\begin{array}{l}\text { Other } \\
\text { allele }\end{array}$ & Mapped gene & MAF & $\begin{array}{l}P_{I N T} \text { with } \\
\text { "active } \\
\text { smoking } \\
\text { status" }\end{array}$ & $\begin{array}{l}P_{I N T} \text { with } \\
\text { "the number } \\
\text { of pack-years" }\end{array}$ & $\begin{array}{l}P_{I N T} \text { with } \\
\text { "years as a } \\
\text { smoker" }\end{array}$ & $\begin{array}{l}P_{I N T} \text { with } \\
\text { "packs } \\
\text { smoked per } \\
\text { day" }\end{array}$ & $\begin{array}{l}P_{I N T} \text { with "hours } \\
\text { as a passive } \\
\text { smoker per } \\
\text { week" }\end{array}$ \\
\hline 2 & rs78529720 & 169777297 & G & $A$ & $A B C B 11$ & 0.289754 & 0.072579 & 0.024974 & 0.019681 & 0.158984 & 0.235235 \\
\hline 6 & rs1012635 & 20675295 & G & $A$ & $C D K A L 1$ & 0.46723 & 0.768481 & 0.923266 & 0.788748 & 0.920955 & 0.140758 \\
\hline 6 & rs35612982 & 20682622 & C & $\mathrm{T}$ & CDKAL1 & 0.346437 & 0.542043 & 0.466119 & 0.466379 & 0.310865 & 0.576941 \\
\hline 7 & rs730497* & 44223721 & A & G & GCK & 0.196822 & 0.024183 & 0.017333 & 0.010552 & 0.008819 & 0.383881 \\
\hline 7 & rs12536382 & 127228728 & C & $\mathrm{T}$ & ARF5 & 0.255785 & 0.192881 & 0.156799 & 0.151725 & 0.371714 & 0.184555 \\
\hline 7 & rs2233580** & 127253550 & $\mathrm{~T}$ & C & PAX4 & 0.106884 & 0.033878 & 0.003083 & 0.010772 & 0.022765 & 0.255327 \\
\hline 8 & rs35859536* & 118191475 & $\mathrm{~T}$ & C & Near SLC30A8 & 0.462279 & 0.662187 & 0.014467 & 0.036535 & 0.117965 & 0.342106 \\
\hline 9 & rs10811661* & 22134094 & C & $\mathrm{T}$ & ------ & 0.416026 & 0.289478 & 0.009493 & 0.092448 & 0.050075 & 0.263594 \\
\hline 10 & rs7896600 & 12255175 & G & C & CDC123 & 0.442577 & 7.25E-07 & $3.94 \mathrm{E}-08$ & 8.48E-07 & 2.02E-09 & 0.384392 \\
\hline 10 & rs1174605899 & 12313728 & A & C & Near CDC123 & 0.489238 & 1.71E-06 & 6.37E-06 & 6.52E-06 & 9.81E-07 & 0.295997 \\
\hline 11 & rs11024175 & 2830565 & $\mathrm{~T}$ & $\mathrm{C}$ & KCNQ1 & 0.406234 & 0.04272 & 0.005488 & 0.012317 & 0.033745 & 0.008735 \\
\hline 17 & rs117948248 & 80759687 & $\mathrm{~T}$ & $\mathrm{C}$ & $T B C D$ & 0.1562 & 0.035671 & 0.000255 & 0.000855 & 0.009197 & 0.083431 \\
\hline
\end{tabular}

Supplemental Table S6 $12 \mathrm{HbA1c}$-associated SNPs discovered by TWB1 ( $P_{S N P}<6.25 \times 10^{-9}$ in model 2)

** rs2233580 was also FG-associated $\left(P_{S N P}<6.25 \times 10^{-9}\right.$ in model 2$)$ and diabetes-associated $\left(P_{S N P}<6.25 \times 10^{-9}\right.$ in model 2$)$.

* rs730497, rs35859536, and rs10811661 were also FG-associated $\left(P_{S N P}<6.25 \times 10^{-9}\right.$ in model 2).

$P_{I N T}$ is the $p$-value of testing $H_{0}: \beta_{I N T}=0$ vs. $H_{1}: \beta_{I N T} \neq 0$ from the following model:

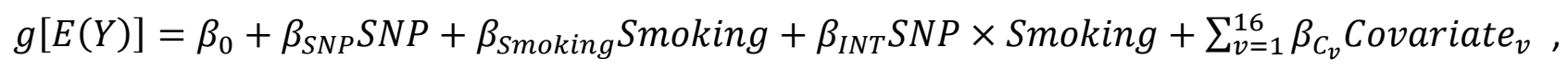

where $Y$ is natural log transformed HbA1c with an identity link $g[\cdot]$, and Smoking is one of the five smoking measurements. 


\begin{tabular}{|c|c|c|c|c|c|c|c|c|c|c|c|}
\hline Chr. & SNP / SNV & Base pair & $\begin{array}{l}\text { Effect } \\
\text { allele }\end{array}$ & $\begin{array}{l}\text { Other } \\
\text { allele }\end{array}$ & Mapped gene & MAF & $\begin{array}{l}P_{I N T} \text { with } \\
\text { "active } \\
\text { smoking } \\
\text { status" }\end{array}$ & $\begin{array}{l}P_{I N T} \text { with } \\
\text { "the number } \\
\text { of pack-years" }\end{array}$ & $\begin{array}{l}P_{I N T} \text { with } \\
\text { "years as a } \\
\text { smoker" }\end{array}$ & $\begin{array}{l}P_{I N T} \text { with } \\
\text { "packs } \\
\text { smoked per } \\
\text { day" }\end{array}$ & $\begin{array}{l}P_{I N T} \text { with "hours } \\
\text { as a passive } \\
\text { smoker per } \\
\text { week" }\end{array}$ \\
\hline 6 & rs3749925 & 20712461 & G & C & $C D K A L 1$ & 0.201348 & 0.317065 & 0.317987 & 0.238203 & 0.326624 & 0.030372 \\
\hline 7 & rs2233580** & 127253550 & $\mathrm{~T}$ & C & PAX4 & 0.106884 & 0.462396 & 0.310362 & 0.381483 & 0.502645 & 0.801273 \\
\hline 7 & rs61342118* & 127761917 & A & C & Near PAX4 & 0.086016 & 0.046082 & 0.159406 & 0.047092 & 0.223836 & 0.823951 \\
\hline 8 & rs13266634*** & 118184783 & $\mathrm{~T}$ & C & SLC30A8 & 0.461692 & 0.111917 & 0.123203 & 0.017727 & 0.298204 & 0.823684 \\
\hline 10 & rs11257655 & 12307894 & C & $\mathbf{T}$ & Near CDC123 & 0.444304 & 0.020900 & 0.109184 & 0.097288 & 0.022917 & 0.042687 \\
\hline 11 & rs163184 & 2847069 & G & $\mathrm{T}$ & KCNQ1 & 0.443249 & 0.131492 & 0.974283 & 0.063704 & 0.945790 & 0.662608 \\
\hline
\end{tabular}

Supplemental Table S7 6 diabetes-associated SNPs discovered by TWB1 $\left(P_{S N P}<6.25 \times 10^{-9}\right)$

** rs2233580 was also FG-associated $\left(P_{S N P}<6.25 \times 10^{-9}\right.$ in model 2) and HbA1c-associated $\left(P_{S N P}<6.25 \times 10^{-9}\right.$ in model 2).

* rs61342118 was also FG-associated $\left(P_{S N P}<6.25 \times 10^{-9}\right.$ in model 2$)$.

*** rs13266634 is also among the 38 diabetes-associated SNPs identified from European GWASs, as listed in the following Tables S18-S20.

$P_{I N T}$ is the $p$-value of testing $H_{0}: \beta_{I N T}=0$ vs. $H_{1}: \beta_{I N T} \neq 0$ from the following model:

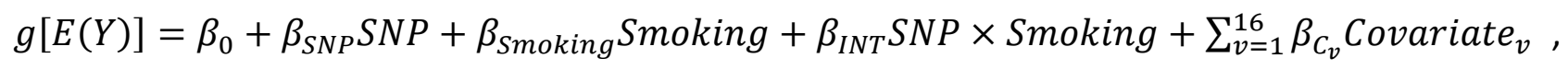

where $Y$ is the diabetes status with a logit link $g[\cdot]$, and Smoking is one of the five smoking measurements. 


\begin{tabular}{|c|c|c|c|c|c|c|}
\hline \multirow[b]{2}{*}{ Explanatory variables in regression model (4) } & \multicolumn{2}{|c|}{ Fasting glucose $(\mathrm{mg} / \mathrm{dL})$} & \multicolumn{2}{|c|}{ HbA1c (\%) } & \multicolumn{2}{|c|}{ Diabetes (dichotomous trait) } \\
\hline & $\begin{array}{c}\text { Percent } \\
\text { change (\%) }\end{array}$ & $p$-value & $\begin{array}{c}\text { Percent } \\
\text { change (\%) }\end{array}$ & $p$-value & Odds ratio & $p$-value \\
\hline $\begin{array}{l}\text { Genetic risk score (GRS) } \\
\text { (z-score standardized) }\end{array}$ & 1.73 & $8.4 \mathrm{E}-172$ & 1.29 & 7.6E-190 & 1.27 & $1.3 \mathrm{E}-35$ \\
\hline $\begin{array}{c}\text { Sex } \\
\text { (1: female vs. 0: male) }\end{array}$ & -3.33 & $2.0 \mathrm{E}-120$ & -0.85 & 7.7E-16 & 0.71 & $6.3 \mathrm{E}-23$ \\
\hline $\begin{array}{l}\text { Age } \\
\text { (in years, continuous variable) }\end{array}$ & 0.36 & 0 & 0.29 & 0 & 1.08 & 0 \\
\hline $\begin{array}{c}\text { Body mass index } \\
\text { (in } \mathrm{kg} / \mathrm{m}^{2} \text {, continuous variable) }\end{array}$ & 0.85 & 0 & 0.74 & 0 & 1.18 & 0 \\
\hline $\begin{array}{c}\text { The number of pack-years } \\
\text { (mean=19.5 pack-years, } s d=17.6 \text { pack-years) }\end{array}$ & 0.03 & 0.19 & 0.03 & 0.03 & 1.01 & 0.002 \\
\hline GRS $\times$ The number of pack-years & 0.06 & $1.6 \mathrm{E}-7$ & 0.02 & 7.3E-4 & 1.002 & 0.15 \\
\hline $\begin{array}{l}\text { Drinking status } \\
\text { (1: yes vs. } 0: \text { no) }\end{array}$ & 1.81 & $8.8 \mathrm{E}-10$ & -1.00 & $2.1 \mathrm{E}-6$ & 0.87 & 0.05 \\
\hline $\begin{array}{l}\text { Regular exercise } \\
\text { (1: yes vs. 0: no) }\end{array}$ & -0.91 & $2.4 \mathrm{E}-12$ & -1.01 & $1.3 \mathrm{E}-26$ & 0.87 & $9.4 \mathrm{E}-6$ \\
\hline $\begin{array}{l}\text { Educational attainment } \\
\text { (a value ranging from } 1 \text { to } 7 \text { ) }\end{array}$ & -0.49 & $2.4 \mathrm{E}-13$ & -0.20 & $3.8 \mathrm{E}-5$ & 0.89 & $2.1 \mathrm{E}-15$ \\
\hline R-square & & & & & & \\
\hline
\end{tabular}

Supplemental Table S8 Results of regression model (4) when the smoking measurement is "The number of pack-years" (including GRS and GRS-smoking interaction) 


\begin{tabular}{|c|c|c|c|c|c|c|}
\hline \multirow[b]{2}{*}{$\begin{array}{l}\text { Explanatory variables in regression } \\
\qquad \text { model (4) }\end{array}$} & \multicolumn{2}{|c|}{ Fasting glucose (mg/dL) } & \multicolumn{2}{|c|}{ HbA1c (\%) } & \multicolumn{2}{|c|}{ Diabetes (dichotomous trait) } \\
\hline & $\begin{array}{c}\text { Percent } \\
\text { change (\%) }\end{array}$ & $p$-value & $\begin{array}{c}\text { Percent } \\
\text { change (\%) }\end{array}$ & $p$-value & Odds ratio & $p$-value \\
\hline $\begin{array}{l}\text { Genetic risk score (GRS) } \\
\text { (z-score standardized) }\end{array}$ & 1.72 & 4.4E-170 & 1.30 & $1.4 \mathrm{E}-190$ & 1.27 & $4.4 \mathrm{E}-35$ \\
\hline $\begin{array}{c}\text { Sex } \\
\text { (1: female vs. 0: male) }\end{array}$ & -3.32 & 4.5E-120 & -0.79 & 3.7E-14 & 0.71 & $1.7 \mathrm{E}-21$ \\
\hline $\begin{array}{c}\text { Age } \\
\text { (in years, continuous variable) }\end{array}$ & 0.36 & 0 & 0.29 & 0 & 1.08 & 0 \\
\hline $\begin{array}{c}\text { Body mass index } \\
\text { (in } \mathrm{kg} / \mathrm{m}^{2} \text {, continuous variable) }\end{array}$ & 0.86 & 0 & 0.74 & 0 & 1.18 & 0 \\
\hline $\begin{array}{c}\text { Years as a smoker } \\
\text { (mean }=26.3 \text { years, } \mathrm{sd}=10.8 \text { years) }\end{array}$ & 0.02 & 0.20 & 0.03 & 0.002 & 1.01 & 0.003 \\
\hline GRS $\times$ Years as a smoker & 0.04 & $8.5 \mathrm{E}-6$ & 0.02 & $2.5 \mathrm{E}-3$ & 1.004 & 0.0048 \\
\hline $\begin{array}{l}\text { Drinking status } \\
\text { (1: yes vs. 0: no) }\end{array}$ & 2.03 & $3.6 \mathrm{E}-11$ & -0.93 & $2.3 \mathrm{E}-5$ & 0.89 & 0.11 \\
\hline $\begin{array}{l}\text { Regular exercise } \\
\text { (1: yes vs. 0: no) }\end{array}$ & -0.92 & $1.3 \mathrm{E}-12$ & -1.01 & $8.6 \mathrm{E}-27$ & 0.86 & $5.1 \mathrm{E}-6$ \\
\hline $\begin{array}{l}\text { Educational attainment } \\
\text { (a value ranging from } 1 \text { to } 7 \text { ) }\end{array}$ & -0.51 & $4.0 \mathrm{E}-14$ & -0.21 & $1.9 \mathrm{E}-5$ & 0.89 & $1.1 \mathrm{E}-15$ \\
\hline R-square & \multicolumn{2}{|c|}{$14.4 \%$} & \multicolumn{2}{|c|}{$15.4 \%$} & \multicolumn{2}{|c|}{$14.0 \%$} \\
\hline
\end{tabular}

Supplemental Table S9 Results of regression model (4) when the smoking measurement is "the number of years as a smoker" (including GRS and GRS-smoking interaction) 


\begin{tabular}{|c|c|c|c|c|c|c|}
\hline \multirow[b]{2}{*}{$\begin{array}{l}\text { Explanatory variables in regression } \\
\text { model (4) }\end{array}$} & \multicolumn{2}{|c|}{ Fasting glucose $(\mathrm{mg} / \mathrm{dL})$} & \multicolumn{2}{|c|}{ HbA1c (\%) } & \multicolumn{2}{|c|}{ Diabetes (dichotomous trait) } \\
\hline & $\begin{array}{l}\text { Percent change } \\
\text { (\%) }\end{array}$ & $p$-value & $\begin{array}{l}\text { Percent change } \\
\text { (\%) }\end{array}$ & $p$-value & Odds ratio & $p$-value \\
\hline $\begin{array}{l}\text { Genetic risk score (GRS) } \\
\text { (z-score standardized) }\end{array}$ & 1.74 & $2.5 \mathrm{E}-172$ & 1.30 & $3.8 \mathrm{E}-190$ & 1.27 & $1.4 \mathrm{E}-35$ \\
\hline $\begin{array}{c}\text { Sex } \\
\text { (1: female vs. 0: male) }\end{array}$ & -3.33 & $2.9 \mathrm{E}-122$ & -0.84 & $6.2 \mathrm{E}-16$ & 0.70 & $1.3 \mathrm{E}-23$ \\
\hline $\begin{array}{c}\text { Age } \\
\text { (in years, continuous variable) }\end{array}$ & 0.36 & 0 & 0.29 & $0^{2}$ & 1.08 & 0 \\
\hline $\begin{array}{c}\text { Body mass index } \\
\text { (in } \mathrm{kg} / \mathrm{m}^{2} \text {, continuous variable) }\end{array}$ & 0.85 & 0 & 0.74 & $0^{2}$ & 1.18 & 0 \\
\hline $\begin{array}{c}\text { Packs smoked per day } \\
\text { (mean }=0.69 \text { packs, } \mathrm{sd}=0.52 \text { packs) }\end{array}$ & 0.89 & 0.08 & 1.02 & 0.006 & 1.38 & 0.01 \\
\hline GRS $\times$ Packs smoked per day & 1.68 & $1.9 \mathrm{E}-7$ & 0.69 & $4.5 \mathrm{E}-4$ & 1.09 & 0.12 \\
\hline $\begin{array}{l}\text { Drinking status } \\
\text { (1: yes vs. 0: no) }\end{array}$ & 1.77 & $2.4 \mathrm{E}-9$ & -1.05 & $9.0 \mathrm{E}-7$ & 0.87 & 0.04 \\
\hline $\begin{array}{l}\text { Regular exercise } \\
\text { (1: yes vs. 0: no) }\end{array}$ & -0.91 & $2.1 \mathrm{E}-12$ & -1.01 & $1.2 \mathrm{E}-26$ & 0.86 & $7.8 \mathrm{E}-6$ \\
\hline $\begin{array}{c}\text { Educational attainment } \\
\text { (a value ranging from } 1 \text { to } 7 \text { ) }\end{array}$ & -0.49 & $5.4 \mathrm{E}-13$ & -0.20 & $6.0 \mathrm{E}-5$ & 0.89 & $3.2 \mathrm{E}-15$ \\
\hline R-square & 14.4 & & 15.4 & & & \\
\hline
\end{tabular}

Supplemental Table S10 Results of regression model (4) when the smoking measurement is "Packs smoked per day" (including GRS and GRS-smoking interaction) 


\begin{tabular}{|c|c|c|c|c|c|c|}
\hline \multirow[b]{2}{*}{ Explanatory variables in regression model (4) } & \multicolumn{2}{|c|}{ Fasting glucose (mg/dL) } & \multicolumn{2}{|c|}{ HbA1c (\%) } & \multicolumn{2}{|c|}{ Diabetes (dichotomous trait) } \\
\hline & $\begin{array}{l}\text { Percent } \\
\text { change }(\%\end{array}$ & $p$-value & Percent change (\%) & $p$-value & Odds ratio & $p$-value \\
\hline $\begin{array}{c}\text { Genetic risk score (GRS) } \\
\text { (z-score standardized) }\end{array}$ & 1.71 & $2.2 \mathrm{E}-167$ & 1.29 & $1.6 \mathrm{E}-188$ & 1.27 & $9.2 \mathrm{E}-36$ \\
\hline $\begin{array}{c}\text { Sex } \\
\text { (1: female vs. 0: male) }\end{array}$ & -3.48 & $1.1 \mathrm{E}-144$ & -1.03 & $6.5 \mathrm{E}-25$ & 0.67 & $1.4 \mathrm{E}-32$ \\
\hline $\begin{array}{c}\text { Age } \\
\text { (in years, continuous variable) }\end{array}$ & 0.36 & 0 & 0.29 & 0 & 1.08 & 0 \\
\hline $\begin{array}{c}\text { Body mass index } \\
\text { (in } \mathrm{kg} / \mathrm{m}^{2} \text {, continuous variable) }\end{array}$ & 0.86 & 0 & 0.74 & 0 & 1.18 & 0 \\
\hline $\begin{array}{l}\text { Hours as a passive smoker per week } \\
\text { (mean }=5.4 \text { hours, } s d=10.6 \text { hours })\end{array}$ & 0.05 & 0.03 & 0.04 & 0.02 & 1.006 & 0.33 \\
\hline GRS $\times$ Hours as a passive smoker per week & 0.02 & 0.25 & -0.007 & 0.58 & 0.996 & 0.30 \\
\hline $\begin{array}{l}\text { Drinking status } \\
\text { (1: yes vs. 0: no) }\end{array}$ & 1.79 & $8.8 \mathrm{E}-11$ & -0.99 & $5.8 \mathrm{E}-7$ & 0.92 & 0.21 \\
\hline $\begin{array}{l}\text { Regular exercise } \\
\text { (1: yes vs. 0: no) }\end{array}$ & -0.95 & $2.0 \mathrm{E}-13$ & -1.06 & 1.7E-29 & 0.85 & $5.9 \mathrm{E}-7$ \\
\hline $\begin{array}{l}\text { Educational attainment } \\
\text { (a value ranging from } 1 \text { to } 7 \text { ) }\end{array}$ & -0.52 & $1.2 \mathrm{E}-14$ & -0.23 & $1.6 \mathrm{E}-6$ & 0.88 & 9.0E-17 \\
\hline R-square & \multicolumn{2}{|c|}{$14.3 \%$} & \multicolumn{2}{|c|}{$15.2 \%$} & \multicolumn{2}{|c|}{$13.9 \%$} \\
\hline
\end{tabular}

Supplemental Table S11 Results of regression model (4) when the smoking measurement is "the number of hours as a passive smoker per week" (including GRS and GRS-smoking interaction) 


\begin{tabular}{|c|c|c|c|c|}
\hline \multirow[b]{2}{*}{$\begin{array}{l}\text { Smoking measurements in regression } \\
\text { model (4) }\end{array}$} & \multicolumn{2}{|c|}{ Fasting glucose (mg/dL) } & \multicolumn{2}{|c|}{$\mathrm{HbA1c}(\%)$} \\
\hline & $\widehat{\phi}_{I N T}$ & $P_{I N T}$ & $\widehat{\phi}_{I N T}$ & $P_{I N T}$ \\
\hline GRS $\times$ Active smoking status & 1.28 & $1.6 \mathrm{E}-4 *$ & 0.0438 & 2.7E-4 * \\
\hline GRS $\times$ The number of pack-years & 0.07 & $2.3 \mathrm{E}-7 *$ & 0.0018 & $7.0 \mathrm{E}-5 *$ \\
\hline GRS $\times$ Years as a smoker & 0.05 & $4.3 \mathrm{E}-5 *$ & 0.0015 & $3.6 \mathrm{E}-4 *$ \\
\hline GRS $\times$ Packs smoked per day & 2.17 & $6.4 \mathrm{E}-7 *$ & 0.0572 & $4.5 \mathrm{E}-5 *$ \\
\hline $\begin{array}{c}\text { GRS } \times \text { Hours as a passive smoker } \\
\text { per week }\end{array}$ & 0.04 & 0.14 & -0.00025 & 0.78 \\
\hline
\end{tabular}

Supplemental Table S12 Results of regression model (4) (without natural log transformation on fasting glucose or HbA1c)

*A total of 15 GRS-smoking interactions were tested in the main manuscript (although the results of dichotomous "diabetes status" were not listed here). A GRS-smoking interaction is significant if $\boldsymbol{P}_{I N T}<\frac{\mathbf{0 . 0 5}}{15}=\mathbf{0 . 0 0 3 3}$. 


\begin{tabular}{|c|c|c|c|c|c|c|}
\hline & \multicolumn{2}{|c|}{ Fasting glucose (mg/dL) } & \multicolumn{2}{|c|}{ HbA1c (\%) } & \multicolumn{2}{|c|}{ Diabetes (dichotomous trait) } \\
\hline $\begin{array}{l}\text { Smoking measurements in regression } \\
\text { model (4) }\end{array}$ & $\begin{array}{l}\text { Percent change } \\
\text { (\%) }\end{array}$ & $P_{I N T}$ & $\begin{array}{l}\text { Percent change } \\
\text { (\%) }\end{array}$ & $P_{I N T}$ & Odds ratio & $P_{I N T}$ \\
\hline GRS $\times$ Active smoking status & 1.22 & $1.8 \mathrm{E}-7 *$ & 0.51 & $0.001 *$ & 1.13 & 0.0063 \\
\hline GRS $\times$ The number of pack-years & 0.07 & $1.0 \mathrm{E}-12 *$ & 0.03 & $3.5 \mathrm{E}-6 *$ & 1.003 & 0.07 \\
\hline GRS $\times$ Years as a smoker & 0.05 & $4.6 \mathrm{E}-9 *$ & 0.02 & 1.7E-4* & 1.004 & $0.0032 *$ \\
\hline GRS $\times$ Packs smoked per day & 2.02 & $2.8 \mathrm{E}-11 *$ & 0.79 & $1.8 \mathrm{E}-5 *$ & 1.08 & 0.11 \\
\hline $\begin{array}{c}\text { GRS } \times \text { Hours as a passive smoker } \\
\text { per week }\end{array}$ & 0.02 & 0.29 & -0.004 & 0.76 & 0.996 & 0.34 \\
\hline
\end{tabular}

Supplemental Table S13 Results of regression model (4) (assess the GRS-smoking interaction without controlling for the other interaction terms)

*A total of 15 GRS-smoking interactions were tested here. A GRS-smoking interaction is significant if $\boldsymbol{P}_{\text {INT }}<\frac{\mathbf{0 . 0 5}}{\mathbf{1 5}}=\mathbf{0 . 0 0 3 3}$. 


\begin{tabular}{|c|c|c|c|c|c|c|c|}
\hline \multirow[b]{2}{*}{$\begin{array}{l}\text { Smoking measurements in regression } \\
\text { model (4) }\end{array}$} & \multirow[b]{2}{*}{ Sex } & \multicolumn{2}{|c|}{ Fasting glucose (mg/dL) } & \multicolumn{2}{|c|}{ HbA1c (\%) } & \multicolumn{2}{|c|}{ Diabetes (dichotomous trait) } \\
\hline & & $\begin{array}{l}\text { Percent change } \\
\text { (\%) }\end{array}$ & $P_{I N T}$ & $\begin{array}{l}\text { Percent change } \\
\text { (\%) }\end{array}$ & $P_{I N T}$ & Odds ratio & $P_{I N T}$ \\
\hline \multirow[t]{2}{*}{ GRS $\times$ Active smoking status } & Males & 1.21 & $2.1 \mathrm{E}-4 *$ & 0.80 & $4.6 \mathrm{E}-4 *$ & 1.18 & 0.0018 \\
\hline & Females & 0.32 & 0.52 & -0.16 & 0.58 & 1.08 & 0.77 \\
\hline \multirow[t]{2}{*}{ GRS $\times$ The number of pack-years } & Males & 0.06 & $3.2 \mathrm{E}-6 *$ & 0.03 & $2.1 \mathrm{E}-4 *$ & 1.003 & 0.033 \\
\hline & Females & -0.03 & 0.37 & -0.02 & 0.23 & 0.99 & 0.25 \\
\hline \multirow[t]{2}{*}{ GRS $\times$ Years as a smoker } & Males & 0.04 & $6.2 \mathrm{E}-5 *$ & 0.03 & $5.1 \mathrm{E}-4 *$ & 1.005 & $0.0013 *$ \\
\hline & Females & -0.00056 & 0.98 & -0.01 & 0.24 & 1.003 & 0.64 \\
\hline \multirow[t]{2}{*}{ GRS $\times$ Packs smoked per day } & Males & 1.86 & $3.6 \mathrm{E}-6 *$ & 0.95 & $1.2 \mathrm{E}-4 *$ & 1.12 & 0.032 \\
\hline & Females & -0.009 & 0.99 & -0.43 & 0.37 & 1.01 & 0.58 \\
\hline \multirow{2}{*}{$\begin{array}{c}\text { GRS } \times \text { Hours as a passive smoker } \\
\text { per week }\end{array}$} & Males & 0.09 & 0.03 & 0.04 & 0.16 & 1.004 & 0.57 \\
\hline & Females & -0.002 & 0.92 & -0.02 & 0.05 & 0.985 & 0.24 \\
\hline
\end{tabular}

Supplemental Table S14 Results of GRS-smoking interactions while stratified by sex

*A total of 30 GRS-smoking interactions were tested here. A GRS-smoking interaction is significant if $\boldsymbol{P}_{I N T}<\frac{\mathbf{0 . 0 5}}{\mathbf{3 0}}=\mathbf{0 . 0 0 1 6}$. 


\begin{tabular}{|c|c|c|c|}
\hline & Overall & Smokers & Non-smokers \\
\hline Total, $n$ & 2,091 & 248 & 1,843 \\
\hline Males, $n(\%)$ & $1,036(49.5)$ & $208(83.9)$ & $828(44.9)$ \\
\hline Age (years), mean (s.d.) & $49.9(11.1)$ & $48.6(10.5)$ & $50.1(11.1)$ \\
\hline BMI $\left(\mathrm{kg} / \mathrm{m}^{2}\right)$, mean (s.d.) & $24.4(3.7)$ & $25.4(3.8)$ & $24.3(3.6)$ \\
\hline Drinking, $n(\%)$ & $145(6.9)$ & $64(25.8)$ & $81(4.4)$ \\
\hline Regular exercise, $n(\%)$ & $924(44.2)$ & $85(34.3)$ & $839(45.5)$ \\
\hline Educational attainment, mean (s.d.) & $5.6(0.9)$ & $5.5(0.8)$ & $5.6(0.9)$ \\
\hline Fasting glucose (mg/dL), mean (s.d.) & $95.9(20.3)$ & $101.5(34.4)$ & $95.1(17.4)$ \\
\hline HbA1c (\%), mean (s.d.) & $5.72(0.74)$ & $5.83(1.01)$ & $5.71(0.69)$ \\
\hline $\mathrm{HbA} 1 \mathrm{c}(\mathrm{mmol} / \mathrm{mol})$, mean (s.d.) & $39(8.1)$ & $40(11.0)$ & $39(7.5)$ \\
\hline Fasting glucose $>126 \mathrm{mg} / \mathrm{dL}, n(\%)$ & $75(3.6)$ & $15(6.0)$ & $60(3.3)$ \\
\hline $\mathrm{HbA} 1 \mathrm{c}>6.5 \%(48 \mathrm{mmol} / \mathrm{mol}), n(\%)$ & $123(5.9)$ & $21(8.5)$ & $102(5.5)$ \\
\hline Subjects with physician-diagnosed diabetes, $n(\%)$ & $86(4.1)$ & $10(4.0)$ & $76(4.1)$ \\
\hline Subjects with diabetes, $n(\%)^{1}$ & $159(7.6)$ & $26(10.5)$ & $133(7.2)$ \\
\hline
\end{tabular}

Supplemental Table S15 Basic characteristics of the 2,091 subjects with blood DNA methylation data

1 Subjects with diabetes included those with physician-diagnosed diabetes, or those having FG $>126 \mathrm{mg} / \mathrm{dL}$ or HbA1c $>6.5 \%(48 \mathrm{mmol} / \mathrm{mol})$ according to the TWB test results. 


\begin{tabular}{|c|c|c|c|c|}
\hline Regression coefficient ( $p$-value) & cg06335123 (in CDC123) & cg26963277 (in KCNQ1) & cg01744331 (in KCNQ1) & cg16556677 (in KCNQ1) \\
\hline \multicolumn{5}{|c|}{ Regressing DNA methylation level on "Packs smoked per day", sex, age, and BMI: } \\
\hline \multicolumn{5}{|c|}{ Negative regression coefficients $=>$ More packs smoked per day, aging, and a larger BMI are associated with decreased levels of DNA methylation at these four sites. } \\
\hline $\begin{array}{l}\text { Packs smoked per day } \\
\text { (mean } \pm \text { sd: } 0.68 \pm 0.51)\end{array}$ & $-0.01349(0.00025)$ & $-0.02033(3.0 \mathrm{E}-17)$ & $-0.02141(4.5 \mathrm{E}-10)$ & $-0.01328(2.7 \mathrm{E}-5)$ \\
\hline $\begin{array}{l}\text { Sex } \\
\text { (1: female vs. 0: male) }\end{array}$ & $0.00577(0.00645)$ & $0.01151(1.1 \mathrm{E}-16)$ & $0.01790(1.8 \mathrm{E}-19)$ & $0.01014(2.7 \mathrm{E}-8)$ \\
\hline $\begin{array}{l}\text { Age } \\
\text { (in years, continuous variable) }\end{array}$ & $-0.00054(3.5 E-9)$ & $-0.00043(2.9 \mathrm{E}-13)$ & $-5.6 \mathrm{E}-4(4.2 \mathrm{E}-11)$ & $-0.00024(1.7 \mathrm{E}-3)$ \\
\hline $\begin{array}{l}\text { Body mass index } \\
\text { (in } \mathrm{kg} / \mathrm{m}^{2} \text {, continuous variable) }\end{array}$ & $-0.00116(4.6 \mathrm{E}-5)$ & $-6.5 E-6(0.97)$ & $-8.7 E-5(0.74)$ & $-0.00021(0.40)$ \\
\hline \multicolumn{5}{|c|}{$\begin{array}{l}\text { "Odds ratio }<1.0 \text { " indicates that an increased DNA methylation level is associated with a lower risk of diabetes, and a decreased DNA methylation level is associated with a } \\
\text { higher risk of diabetes. }\end{array}$} \\
\hline Odds ratio ( $p$-value) & $0.0082(0.0413)$ & $0.0009(0.0045)$ & $0.0075(0.0118)$ & $0.0700(0.2083)$ \\
\hline
\end{tabular}

Supplemental Table S16 Analysis results of blood DNA methylation data 


\begin{tabular}{|c|c|c|c|c|c|c|c|c|c|c|c|}
\hline SNP rsID & Chr & Position & $\begin{array}{l}\text { Effect } \\
\text { allele }\end{array}$ & $\begin{array}{l}\text { Other } \\
\text { allele }\end{array}$ & $\begin{array}{l}\text { Mapped } \\
\text { gene }\end{array}$ & MAF & $\begin{array}{l}P_{I N T} \text { with } \\
\text { "active smoking } \\
\text { status" } 1\end{array}$ & $\begin{array}{l}P_{I N T} \text { with "the } \\
\text { number of } \\
\text { pack-years" } 1\end{array}$ & $\begin{array}{l}P_{I N T} \text { with } \\
\text { "years as a } \\
\text { smoker" } 1\end{array}$ & $\begin{array}{l}P_{I N T} \text { with } \\
\text { "packs smoked } \\
\text { per day" } 1\end{array}$ & $\begin{array}{l}P_{I N T} \text { with "hours } \\
\text { as a passive smoker } \\
\text { per week" } 1\end{array}$ \\
\hline
\end{tabular}

\section{SNP-smoking interaction analysis on natural log transformed fasting glucose:}

\begin{tabular}{|c|c|c|c|c|c|c|c|c|c|c|c|}
\hline rs1444261 & 2 & 55354466 & C & $\mathrm{T}$ & C2orf63 & 0.062213 & 0.647064 & 0.067076 & 0.340325 & 0.191402 & 0.263129 \\
\hline rs1801232 & 10 & 16870912 & $\mathrm{~T}$ & G & CUBN & 0.156081 & 0.294669 & 0.759270 & 0.453210 & 0.882073 & 0.960374 \\
\hline rs4132670 & 10 & 114767771 & A & G & TCF7L2 & 0.005402 & 0.120335 & 0.915558 & 0.287107 & 0.839836 & 0.019209 \\
\hline rs12243326 & 10 & 114788815 & C & $\mathrm{T}$ & TCF7L2 & 0.005368 & 0.102001 & 0.931361 & 0.261229 & 0.850150 & 0.019397 \\
\hline rs140637 & 15 & 48766855 & G & $A$ & FBN1 & 0.000009 & & $t$ is $t$ & in Taiwar & & \\
\hline
\end{tabular}

SNP-smoking interaction analysis on natural log transformed HbA1c:

\begin{tabular}{|c|c|c|c|c|c|c|c|c|c|c|c|}
\hline rs1444261 & 2 & 55354466 & C & $\mathrm{T}$ & C2orf63 & 0.062213 & 0.515846 & 0.132088 & 0.951349 & 0.380867 & 0.728838 \\
\hline rs1801232 & 10 & 16870912 & $\mathrm{~T}$ & G & CUBN & 0.156081 & 0.057120 & 0.113832 & 0.064861 & 0.167234 & 0.043065 \\
\hline rs4132670 & 10 & 114767771 & A & G & TCF7L2 & 0.005402 & 0.029237 & 0.654357 & 0.155104 & 0.513931 & 0.095402 \\
\hline rs12243326 & 10 & 114788815 & C & $\mathrm{T}$ & TCF7L2 & 0.005368 & 0.025216 & 0.703848 & 0.154910 & 0.557736 & 0.097694 \\
\hline rs140637 & 15 & 48766855 & G & $A$ & FBN1 & 0.000009 & lts hecau & ant is to & in Taiwan & & \\
\hline
\end{tabular}

SNP-smoking interaction analysis on diabetes status:

\begin{tabular}{|c|c|c|c|c|c|c|c|c|c|c|c|}
\hline rs1444261 & 2 & 55354466 & C & $\mathrm{T}$ & C2orf63 & 0.062213 & 0.759288 & 0.113310 & 0.515076 & 0.321233 & 0.624802 \\
\hline rs1801232 & 10 & 16870912 & $\mathrm{~T}$ & G & CUBN & 0.156081 & 0.396722 & 0.713981 & 0.620736 & 0.664798 & 0.733262 \\
\hline rs4132670 & 10 & 114767771 & $A$ & G & TCF7L2 & 0.005402 & 0.014853 & 0.579142 & 0.079367 & 0.371905 & 0.077710 \\
\hline rs12243326 & 10 & 114788815 & $\mathrm{C}$ & $\mathrm{T}$ & TCF7L2 & 0.005368 & 0.012956 & 0.573007 & 0.074901 & 0.368543 & 0.079007 \\
\hline rs140637 & 15 & 48766855 & G & A & FBN1 & 0.000009 & \multicolumn{5}{|c|}{ No results because this variant is too rare in Taiwan Biobank. } \\
\hline
\end{tabular}

Supplemental Table S17 The 5 SNP-smoking interactions on diabetes identified from subjects of European and African ancestry

${ }^{1} P_{I N T}$ is the $p$-value of testing $H_{0}: \beta_{I N T}=0$ vs. $H_{1}: \beta_{I N T} \neq 0$ from the following model:

$$
g[E(Y)]=\beta_{0}+\beta_{S N P} S N P+\beta_{\text {Smoking }} \text { Smoking }+\beta_{I N T} S N P \times \text { Smoking }+\sum_{v=1}^{16} \beta_{C_{v}} \text { Covariate }_{v},
$$

where $Y$ is natural log transformed FG or HbA1c with an identity link $g[\cdot]$, or the diabetes status with a logit link $g[\cdot]$. Smoking is one of the five smoking measurements. $P_{I N T}<0.05$ was highlighted in yellow. 


\begin{tabular}{|c|c|c|c|c|c|c|c|c|c|c|c|}
\hline SNP rsID & Chr & Position & $\begin{array}{l}\text { Effect } \\
\text { allele }\end{array}$ & $\begin{array}{l}\text { Other } \\
\text { allele }\end{array}$ & Mapped gene & MAF & $\begin{array}{l}P_{I N T} \text { with } \\
\text { "active } \\
\text { smoking } \\
\text { status" } 1\end{array}$ & $\begin{array}{l}P_{I N T} \text { with } \\
\text { "the number } \\
\text { of } \\
\text { pack-years" } 1\end{array}$ & $\begin{array}{l}P_{I N T} \text { with } \\
\text { "years as a } \\
\text { smoker" } 1\end{array}$ & $\begin{array}{l}P_{I N T} \text { with } \\
\text { "packs smoked } \\
\text { per day" } 1\end{array}$ & $\begin{array}{l}P_{I N T} \text { with "hours } \\
\text { as a passive } \\
\text { smoker per } \\
\text { week" } 1^{1}\end{array}$ \\
\hline rs10923931 & 1 & 120517959 & $\mathrm{~T}$ & G & NOTCH2 & 0.0266 & 0.497297 & 0.758139 & 0.733951 & 0.657158 & 0.500067 \\
\hline rs340874 & 1 & 214159256 & $\mathrm{C}$ & $\mathrm{T}$ & PROX1 & 0.3866 & 0.34053 & 0.047393 & 0.51152 & 0.050668 & 0.93393 \\
\hline rs780094 & 2 & 27741237 & C & $\mathrm{T}$ & $G C K R$ & 0.4805 & 0.663052 & 0.885067 & 0.922033 & 0.630088 & 0.066742 \\
\hline rs7578597 & 2 & 43732823 & $\mathrm{~T}$ & C & THADA & 0.0052 & 0.073969 & 0.215989 & 0.09459 & 0.147412 & 0.535595 \\
\hline rs243021 & 2 & 60584819 & $A$ & G & -------- & 0.3388 & 0.140963 & 0.195474 & 0.160076 & 0.144135 & 0.580446 \\
\hline rs13389219 & 2 & 165528876 & $\mathrm{C}$ & $\mathrm{T}$ & COBLL1 & 0.1028 & 0.110077 & 0.110058 & 0.15842 & 0.037917 & 0.732636 \\
\hline rs7578326 & 2 & 227020653 & $A$ & G & LOC646736 & 0.1510 & 0.191067 & 0.483716 & 0.588048 & 0.158477 & 0.752026 \\
\hline rs17036101 & 3 & 12277845 & G & $A$ & -------- & 0.0339 & 0.81851 & 0.894187 & 0.919896 & 0.90745 & 0.003235 \\
\hline rs4607103 & 3 & 64711904 & C & $\mathrm{T}$ & ADAMTS9-AS2 & 0.3451 & 0.782297 & 0.930636 & 0.977063 & 0.57864 & 0.245092 \\
\hline rs11708067 & 3 & 123065778 & A & G & $A D C Y 5$ & 0.0006 & 0.32134 & 0.603048 & 0.565434 & 0.513541 & 0.469027 \\
\hline rs459193 & 5 & 55806751 & G & $A$ & C5orf67 & 0.4687 & 0.559281 & 0.342269 & 0.676071 & 0.138188 & 0.640302 \\
\hline rs4457053 & 5 & 76424949 & G & $A$ & ZBED3-AS1 & 0.0559 & 0.038005 & 0.000692 & 0.008547 & 0.002559 & 0.145399 \\
\hline rs9472138 & 6 & 43811762 & $\mathrm{~T}$ & $\mathrm{C}$ & LOC107986598 & 0.1390 & 0.045065 & 0.000116 & 0.003241 & 0.002329 & 0.870623 \\
\hline rs2191349 & 7 & 15064309 & $\mathrm{~T}$ & G & -------- & 0.3098 & 0.59694 & 0.221766 & 0.265209 & 0.342553 & 0.218164 \\
\hline rs864745 & 7 & 28180556 & $\mathrm{~T}$ & $\mathrm{C}$ & $J A Z F 1$ & 0.2134 & 0.438802 & 0.559807 & 0.724187 & 0.456061 & 0.21298 \\
\hline rs972283 & 7 & 130466854 & G & $A$ & LOC105375508 & 0.3136 & 0.058095 & 0.018156 & 0.073993 & 0.004794 & 0.562678 \\
\hline rs516946 & 8 & 41519248 & $\mathrm{C}$ & $\mathrm{T}$ & ANK1 & 0.1267 & 0.346076 & 0.103098 & 0.048341 & 0.561153 & 0.215356 \\
\hline rs896854 & 8 & 95960511 & $\mathrm{~T}$ & $\mathrm{C}$ & TP53INP1 & 0.2746 & 0.858037 & 0.676124 & 0.94125 & 0.504252 & 0.852747 \\
\hline rs13266634*** & 8 & 118184783 & C & $\mathrm{T}$ & $S L C 30 A 8^{2}$ & 0.4617 & 0.648976 & 0.065385 & 0.09736 & 0.312004 & 0.127494 \\
\hline rs13292136 & 9 & 81952128 & C & $\mathrm{T}$ & -------- & 0.0870 & 0.357974 & 0.818348 & 0.917207 & 0.170779 & 0.283483 \\
\hline rs2796441 & 9 & 84308948 & G & $A$ & LOC101927502 & 0.3837 & 0.068336 & 0.11528 & 0.088995 & 0.048266 & 0.796523 \\
\hline rs12779790 & 10 & 12328010 & G & A & Near CDC123 ${ }^{2}$ & 0.1824 & 0.224513 & 0.112216 & 0.311603 & 0.049073 & 0.95917 \\
\hline rs12571751 & 10 & 80942631 & $A$ & G & ZMIZ1 & 0.4227 & 0.565342 & 0.792829 & 0.210464 & 0.87981 & 0.007788 \\
\hline
\end{tabular}




\begin{tabular}{|c|c|c|c|c|c|c|c|c|c|c|c|}
\hline SNP rsID & Chr & Position & $\begin{array}{l}\text { Effect } \\
\text { allele }\end{array}$ & $\begin{array}{l}\text { Other } \\
\text { allele }\end{array}$ & Mapped gene & MAF & $\begin{array}{l}P_{I N T} \text { with } \\
\text { "active } \\
\text { smoking } \\
\text { status" } 1\end{array}$ & $\begin{array}{l}P_{I N T} \text { with } \\
\text { "the number } \\
\text { of } \\
\text { pack-years" } 1\end{array}$ & $\begin{array}{l}P_{I N T} \text { with } \\
\text { "years as a } \\
\text { smoker" } 1\end{array}$ & $\begin{array}{l}P_{I N T} \text { with } \\
\text { "packs smoked } \\
\text { per day" } 1\end{array}$ & $\begin{array}{l}P_{I N T} \text { with "hours } \\
\text { as a passive } \\
\text { smoker per } \\
\text { week" } 1^{1}\end{array}$ \\
\hline rs7903146 & 10 & 114758349 & $\mathrm{~T}$ & C & TCF7L2 & 0.0236 & 0.53338 & 0.031689 & 0.226831 & 0.42343 & 0.276035 \\
\hline rs231362 & 11 & 2691471 & G & $A$ & KCNQ1 ${ }^{2}$ & 0.0958 & 0.808066 & 0.602967 & 0.846658 & 0.583126 & 0.386619 \\
\hline rs1552224 & 11 & 72433098 & $A$ & C & ARAP1 & 0.0663 & 0.440084 & 0.877019 & 0.603174 & 0.952758 & 0.965577 \\
\hline rs1387153 & 11 & 92673828 & $\mathrm{~T}$ & C & -------- & 0.4563 & 0.864188 & 0.134995 & 0.22974 & 0.561622 & 0.216203 \\
\hline rs10842994 & 12 & 27965150 & C & $\mathrm{T}$ & LOC105369709 & 0.2070 & 0.450971 & 0.411314 & 0.919561 & 0.39939 & 0.781914 \\
\hline rs1153188 & 12 & 55098996 & $A$ & $\mathrm{~T}$ & -------- & 0.0145 & 0.716316 & 0.125885 & 0.732642 & 0.167496 & 0.756387 \\
\hline rs1531343 & 12 & 66174894 & $\mathrm{C}$ & G & RPSAP52 & 0.1091 & 0.267408 & 0.265706 & 0.219831 & 0.350382 & 0.331831 \\
\hline rs7961581 & 12 & 71663102 & C & $\mathrm{T}$ & -------- & 0.2287 & 0.991809 & 0.401535 & 0.701329 & 0.388973 & 0.851531 \\
\hline rs7957197 & 12 & 121460686 & $\mathrm{~T}$ & $A$ & $O A S L$ & 0.0006 & 0.977098 & 0.921786 & 0.985963 & 0.901942 & 0.776688 \\
\hline rs7177055 & 15 & 77832762 & $A$ & G & -------- & 0.3341 & 0.819691 & 0.999054 & 0.565509 & 0.759913 & 0.64185 \\
\hline rs11634397 & 15 & 80432222 & G & $A$ & -------- & 0.0836 & 0.225901 & 0.008178 & 0.115465 & 0.024652 & 0.594659 \\
\hline rs8042680 & 15 & 91521337 & $A$ & $\mathrm{C}$ & PRC1 & 0.0029 & 0.558726 & 0.499235 & 0.410369 & 0.438919 & 0.746225 \\
\hline rs7202877 & 16 & 75247245 & $\mathrm{~T}$ & G & -------- & 0.2050 & 0.927582 & 0.184688 & 0.84189 & 0.248119 & 0.882796 \\
\hline rs12970134 & 18 & 57884750 & $A$ & G & -------- & 0.1738 & 0.435106 & 0.12862 & 0.555369 & 0.117711 & 0.309806 \\
\hline rs10401969 & 19 & 19407718 & C & $\mathrm{T}$ & SUGP1 & 0.0965 & 0.214083 & 0.028897 & 0.223583 & 0.022962 & 0.408376 \\
\hline
\end{tabular}

Supplemental Table S18 (The 38 diabetes-associated SNPs identified from European GWASs) SNP-smoking interaction analysis on natural log

transformed fasting glucose

${ }^{1} P_{I N T}$ is the $p$-value of testing $H_{0}: \beta_{I N T}=0$ vs. $H_{1}: \beta_{I N T} \neq 0$ from the following model:

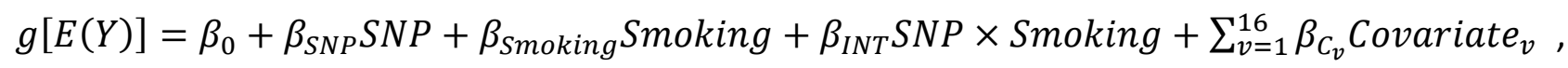

where $Y$ is natural log transformed fasting glucose with an identity link $g[\cdot]$, and Smoking is one of the five smoking measurements. $P_{I N T}<0.05$ 
was highlighted in yellow.

${ }^{2}$ CDC123, KCNQ1, and SLC30A8 were also associated with FG/HbA1c/diabetes according to our discovery cohort (TWB1), as listed in the above Tables S5-S7.

*** SNP rs13266634 (in the SLC30A8 gene) is also among the 6 diabetes-associated SNPs discovered by TWB1, as listed in the above Table S7. 


\begin{tabular}{|c|c|c|c|c|c|c|c|c|c|c|c|}
\hline SNP rsID & Chr & Position & $\begin{array}{l}\text { Effect } \\
\text { allele }\end{array}$ & $\begin{array}{l}\text { Other } \\
\text { allele }\end{array}$ & Mapped gene & MAF & $\begin{array}{l}P_{I N T} \text { with } \\
\text { "active } \\
\text { smoking } \\
\text { status" } 1\end{array}$ & $\begin{array}{l}P_{I N T} \text { with } \\
\text { "the number } \\
\text { of } \\
\text { pack-years" } 1\end{array}$ & $\begin{array}{l}P_{I N T} \text { with } \\
\text { "years as a } \\
\text { smoker" } 1\end{array}$ & $\begin{array}{l}P_{I N T} \text { with } \\
\text { "packs smoked } \\
\text { per day" } 1\end{array}$ & $\begin{array}{l}P_{I N T} \text { with "hours } \\
\text { as a passive } \\
\text { smoker per } \\
\text { week" }^{1}\end{array}$ \\
\hline rs10923931 & 1 & 120517959 & $\mathrm{~T}$ & G & NOTCH2 & 0.0266 & 0.098816 & 0.974414 & 0.081733 & 0.727306 & 0.80535 \\
\hline rs340874 & 1 & 214159256 & $\mathrm{C}$ & $\mathrm{T}$ & PROX1 & 0.3866 & 0.615122 & 0.745585 & 0.642277 & 0.673305 & 0.299826 \\
\hline rs780094 & 2 & 27741237 & C & $\mathrm{T}$ & GCKR & 0.4805 & 0.027893 & 0.060585 & 0.002821 & 0.162519 & 0.273771 \\
\hline rs7578597 & 2 & 43732823 & $\mathrm{~T}$ & C & THADA & 0.0052 & 0.020788 & 0.014977 & 0.003526 & 0.037148 & 0.610847 \\
\hline rs243021 & 2 & 60584819 & $A$ & G & -------- & 0.3388 & 0.991047 & 0.796397 & 0.935671 & 0.879511 & 0.626304 \\
\hline rs13389219 & 2 & 165528876 & $\mathrm{C}$ & $\mathrm{T}$ & COBLL1 & 0.1028 & 0.170474 & 0.64622 & 0.371401 & 0.287757 & 0.697176 \\
\hline rs7578326 & 2 & 227020653 & $A$ & G & LOC646736 & 0.1510 & 0.218471 & 0.324675 & 0.416461 & 0.252683 & 0.953437 \\
\hline rs17036101 & 3 & 12277845 & G & $A$ & -------- & 0.0339 & 0.606802 & 0.873054 & 0.631549 & 0.953848 & 0.016144 \\
\hline rs4607103 & 3 & 64711904 & C & $\mathrm{T}$ & ADAMTS9-AS2 & 0.3451 & 0.695618 & 0.764223 & 0.598439 & 0.948124 & 0.14818 \\
\hline rs11708067 & 3 & 123065778 & $A$ & G & $A D C Y 5$ & 0.0006 & 0.48117 & 0.819303 & 0.724267 & 0.742881 & 0.628109 \\
\hline rs459193 & 5 & 55806751 & G & $A$ & C5orf67 & 0.4687 & 0.416742 & 0.981739 & 0.623708 & 0.547031 & 0.625168 \\
\hline rs4457053 & 5 & 76424949 & G & $A$ & ZBED3-AS1 & 0.0559 & 0.516869 & 0.101914 & 0.282282 & 0.133313 & 0.046199 \\
\hline rs9472138 & 6 & 43811762 & $\mathrm{~T}$ & C & LOC107986598 & 0.1390 & 0.070302 & 0.000355 & 0.002948 & 0.008385 & 0.556876 \\
\hline rs2191349 & 7 & 15064309 & $\mathrm{~T}$ & G & -------- & 0.3098 & 0.372583 & 0.494297 & 0.742857 & 0.642247 & 0.404233 \\
\hline rs864745 & 7 & 28180556 & $\mathrm{~T}$ & C & JAZF1 & 0.2134 & 0.724627 & 0.993206 & 0.483087 & 0.986243 & 0.464115 \\
\hline rs972283 & 7 & 130466854 & G & $A$ & LOC105375508 & 0.3136 & 0.031582 & 0.055947 & 0.044097 & 0.01254 & 0.438507 \\
\hline rs516946 & 8 & 41519248 & $\mathrm{C}$ & $\mathrm{T}$ & ANK1 & 0.1267 & 0.424793 & 0.993553 & 0.798159 & 0.39221 & 0.093636 \\
\hline rs896854 & 8 & 95960511 & $\mathrm{~T}$ & $\mathrm{C}$ & TP53INP1 & 0.2746 & 0.95641 & 0.831707 & 0.956592 & 0.907038 & 0.517093 \\
\hline rs13266634*** & 8 & 118184783 & C & $\mathrm{T}$ & $S \angle C 30 A 8^{2}$ & 0.4617 & 0.658035 & 0.014351 & 0.036456 & 0.117095 & 0.388746 \\
\hline rs13292136 & 9 & 81952128 & C & $\mathrm{T}$ & -------- & 0.0870 & 0.473158 & 0.264458 & 0.149703 & 0.929171 & 0.036055 \\
\hline rs2796441 & 9 & 84308948 & G & $A$ & LOC101927502 & 0.3837 & 0.551078 & 0.908062 & 0.618745 & 0.641946 & 0.50278 \\
\hline rs12779790 & 10 & 12328010 & $\mathbf{G}$ & A & Near $C D C 123^{2}$ & 0.1824 & 0.00151 & 0.000427 & 0.003391 & 8.91E-05 & 0.918916 \\
\hline rs12571751 & 10 & 80942631 & $A$ & G & ZMIZ1 & 0.4227 & 0.79652 & 0.933041 & 0.355454 & 0.858318 & 0.076816 \\
\hline
\end{tabular}




\begin{tabular}{|c|c|c|c|c|c|c|c|c|c|c|c|}
\hline SNP rsID & Chr & Position & $\begin{array}{l}\text { Effect } \\
\text { allele }\end{array}$ & $\begin{array}{l}\text { Other } \\
\text { allele }\end{array}$ & Mapped gene & MAF & $\begin{array}{l}P_{I N T} \text { with } \\
\text { "active } \\
\text { smoking } \\
\text { status" } 1\end{array}$ & $\begin{array}{l}P_{I N T} \text { with } \\
\text { "the number } \\
\text { of } \\
\text { pack-years" } 1\end{array}$ & $\begin{array}{l}P_{I N T} \text { with } \\
\text { "years as a } \\
\text { smoker" } 1\end{array}$ & $\begin{array}{l}P_{I N T} \text { with } \\
\text { "packs smoked } \\
\text { per day" } 1\end{array}$ & $\begin{array}{l}P_{I N T} \text { with "hours } \\
\text { as a passive } \\
\text { smoker per } \\
\text { week" }^{1}\end{array}$ \\
\hline rs7903146 & 10 & 114758349 & $\mathrm{~T}$ & C & TCF7L2 & 0.0236 & 0.117409 & 0.02642 & 0.07501 & 0.267786 & 0.915459 \\
\hline rs231362 & 11 & 2691471 & G & $A$ & $K C N Q 1{ }^{2}$ & 0.0958 & 0.683558 & 0.382904 & 0.792207 & 0.505264 & 0.827145 \\
\hline rs1552224 & 11 & 72433098 & $A$ & $\mathrm{C}$ & ARAP1 & 0.0663 & 0.290744 & 0.498728 & 0.19708 & 0.798587 & 0.156948 \\
\hline rs1387153 & 11 & 92673828 & $\mathrm{~T}$ & C & -------- & 0.4563 & 0.648617 & 0.449784 & 0.496341 & 0.886364 & 0.149319 \\
\hline rs10842994 & 12 & 27965150 & C & $\mathrm{T}$ & LOC105369709 & 0.2070 & 0.560089 & 0.777197 & 0.098758 & 0.941118 & 0.493545 \\
\hline rs1153188 & 12 & 55098996 & $A$ & $\mathrm{~T}$ & ------- & 0.0145 & 0.195607 & 0.853368 & 0.282086 & 0.702558 & 0.634745 \\
\hline rs1531343 & 12 & 66174894 & C & G & RPSAP52 & 0.1091 & 0.375011 & 0.090797 & 0.140622 & 0.195879 & 0.613751 \\
\hline rs7961581 & 12 & 71663102 & C & $\mathrm{T}$ & ------- & 0.2287 & 0.713713 & 0.839775 & 0.401286 & 0.549239 & 0.160869 \\
\hline rs7957197 & 12 & 121460686 & $\mathrm{~T}$ & $A$ & OASL & 0.0006 & 0.915054 & 0.832918 & 0.936515 & 0.864064 & 0.625215 \\
\hline rs7177055 & 15 & 77832762 & $A$ & G & ------- & 0.3341 & 0.678558 & 0.183532 & 0.61204 & 0.163101 & 0.404036 \\
\hline rs11634397 & 15 & 80432222 & G & $A$ & ------- & 0.0836 & 0.983663 & 0.072363 & 0.703859 & 0.123287 & 0.626227 \\
\hline rs8042680 & 15 & 91521337 & $A$ & $\mathrm{C}$ & $P R C 1$ & 0.0029 & 0.124564 & 0.356929 & 0.028107 & 0.602057 & 0.406163 \\
\hline rs7202877 & 16 & 75247245 & $T$ & G & -------- & 0.2050 & 0.953117 & 0.106242 & 0.784282 & 0.113571 & 0.39607 \\
\hline rs12970134 & 18 & 57884750 & A & G & -------- & 0.1738 & 0.002284 & 0.001549 & 0.0032 & 0.001553 & 0.353752 \\
\hline rs10401969 & 19 & 19407718 & C & $T$ & SUGP1 & 0.0965 & 0.618241 & 0.366786 & 0.901497 & 0.25566 & 0.786735 \\
\hline
\end{tabular}

Supplemental Table S19 (The 38 diabetes-associated SNPs identified from European GWASs) SNP-smoking interaction analysis on natural log transformed HbA1c

${ }^{1} P_{I N T}$ is the $p$-value of testing $H_{0}: \beta_{I N T}=0$ vs. $H_{1}: \beta_{I N T} \neq 0$ from the following model:

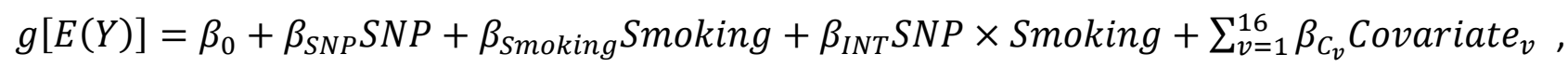

where $Y$ is natural log transformed HbA1c with an identity link $g[\cdot]$, and Smoking is one of the five smoking measurements. $P_{I N T}<0.05$ was 
highlighted in yellow.

${ }^{2}$ CDC123, KCNQ1, and SLC30A8 were also associated with FG/HbA1c/diabetes according to our discovery cohort (TWB1), as listed in the above Tables S5-S7.

*** SNP rs13266634 (in the SLC30A8 gene) is also among the 6 diabetes-associated SNPs discovered by TWB1, as listed in the above Table S7. 


\begin{tabular}{|c|c|c|c|c|c|c|c|c|c|c|c|}
\hline SNP rsID & Chr & Position & $\begin{array}{l}\text { Effect } \\
\text { allele }\end{array}$ & $\begin{array}{l}\text { Other } \\
\text { allele }\end{array}$ & Mapped gene & MAF & $\begin{array}{l}P_{I N T} \text { with } \\
\text { "active } \\
\text { smoking } \\
\text { status" } 1\end{array}$ & $\begin{array}{l}P_{I N T} \text { with } \\
\text { "the number } \\
\text { of } \\
\text { pack-years" } 1\end{array}$ & $\begin{array}{l}P_{I N T} \text { with } \\
\text { "years as a } \\
\text { smoker" } 1\end{array}$ & $\begin{array}{l}P_{I N T} \text { with } \\
\text { "packs smoked } \\
\text { per day" } 1\end{array}$ & $\begin{array}{l}P_{I N T} \text { with "hours } \\
\text { as a passive } \\
\text { smoker per } \\
\text { week" } 1^{1}\end{array}$ \\
\hline rs10923931 & 1 & 120517959 & $\mathrm{~T}$ & G & NOTCH2 & 0.0266 & 0.179347 & 0.720227 & 0.427992 & 0.975307 & 0.787074 \\
\hline rs340874 & 1 & 214159256 & $\mathrm{C}$ & $\mathrm{T}$ & PROX1 & 0.3866 & 0.80797 & 0.717182 & 0.970264 & 0.742546 & 0.880438 \\
\hline rs780094 & 2 & 27741237 & $\mathrm{C}$ & $\mathrm{T}$ & GCKR & 0.4805 & 0.177751 & 0.10087 & 0.052343 & 0.249735 & 0.270362 \\
\hline rs7578597 & 2 & 43732823 & $\mathrm{~T}$ & C & THADA & 0.0052 & 0.045987 & 0.225273 & 0.054377 & 0.26392 & 0.548494 \\
\hline rs243021 & 2 & 60584819 & $A$ & G & -------- & 0.3388 & 0.961979 & 0.309409 & 0.657621 & 0.291143 & 0.837461 \\
\hline rs13389219 & 2 & 165528876 & $\mathrm{C}$ & $\mathrm{T}$ & COBLL1 & 0.1028 & 0.896581 & 0.324198 & 0.456718 & 0.80187 & 0.622696 \\
\hline rs7578326 & 2 & 227020653 & $A$ & G & LOC646736 & 0.1510 & 0.401498 & 0.587061 & 0.648855 & 0.516609 & 0.425919 \\
\hline rs17036101 & 3 & 12277845 & G & $A$ & -------- & 0.0339 & 0.333526 & 0.614036 & 0.399858 & 0.56662 & 0.059133 \\
\hline rs4607103 & 3 & 64711904 & $\mathrm{C}$ & $\mathrm{T}$ & ADAMTS9-AS2 & 0.3451 & 0.724647 & 0.745187 & 0.746284 & 0.727742 & 0.329993 \\
\hline rs11708067 & 3 & 123065778 & A & G & $A D C Y 5$ & 0.0006 & 0.926012 & 0.920493 & 0.932579 & 0.913228 & 0.948399 \\
\hline rs459193 & 5 & 55806751 & G & $A$ & C5orf67 & 0.4687 & 0.633853 & 0.182762 & 0.48204 & 0.151906 & 0.462609 \\
\hline rs4457053 & 5 & 76424949 & G & $A$ & ZBED3-AS1 & 0.0559 & 0.283105 & 0.248024 & 0.128189 & 0.354533 & 0.080364 \\
\hline rs9472138 & 6 & 43811762 & $\mathrm{~T}$ & $\mathrm{C}$ & LOC107986598 & 0.1390 & 0.382594 & 0.592256 & 0.524271 & 0.604028 & 0.55964 \\
\hline rs2191349 & 7 & 15064309 & $\mathrm{~T}$ & G & -------- & 0.3098 & 0.89061 & 0.836876 & 0.706284 & 0.8688 & 0.182585 \\
\hline rs864745 & 7 & 28180556 & $\mathrm{~T}$ & $\mathrm{C}$ & $J A Z F 1$ & 0.2134 & 0.723531 & 0.41901 & 0.661041 & 0.577499 & 0.904833 \\
\hline rs972283 & 7 & 130466854 & G & $A$ & LOC105375508 & 0.3136 & 0.02176 & 0.061506 & 0.039392 & 0.023175 & 0.786707 \\
\hline rs516946 & 8 & 41519248 & $\mathrm{C}$ & $\mathrm{T}$ & ANK1 & 0.1267 & 0.1877 & 0.554055 & 0.357718 & 0.348253 & 0.823944 \\
\hline rs896854 & 8 & 95960511 & $\mathrm{~T}$ & $\mathrm{C}$ & TP53INP1 & 0.2746 & 0.95521 & 0.979715 & 0.963165 & 0.857873 & 0.616662 \\
\hline rs13266634*** & 8 & 118184783 & C & $\mathrm{T}$ & SLC3OA ${ }^{2}$ & 0.4617 & 0.111917 & 0.123203 & 0.017727 & 0.298204 & 0.823684 \\
\hline rs13292136 & 9 & 81952128 & C & $\mathrm{T}$ & -------- & 0.0870 & 0.716811 & 0.682723 & 0.58285 & 0.992995 & 0.31485 \\
\hline rs2796441 & 9 & 84308948 & G & $A$ & LOC101927502 & 0.3837 & 0.705856 & 0.426133 & 0.540604 & 0.84844 & 0.668982 \\
\hline rs12779790 & 10 & 12328010 & G & A & Near $C D C 123^{2}$ & 0.1824 & 0.031129 & 0.080318 & 0.134924 & 0.017302 & 0.675323 \\
\hline rs12571751 & 10 & 80942631 & $A$ & G & ZMIZ1 & 0.4227 & 0.298901 & 0.737266 & 0.481161 & 0.769763 & 0.401545 \\
\hline
\end{tabular}




\begin{tabular}{|c|c|c|c|c|c|c|c|c|c|c|c|}
\hline SNP rsID & Chr & Position & $\begin{array}{l}\text { Effect } \\
\text { allele }\end{array}$ & $\begin{array}{l}\text { Other } \\
\text { allele }\end{array}$ & Mapped gene & MAF & $\begin{array}{l}P_{I N T} \text { with } \\
\text { "active } \\
\text { smoking } \\
\text { status" } 1\end{array}$ & $\begin{array}{l}P_{I N T} \text { with } \\
\text { "the number } \\
\text { of } \\
\text { pack-years" } 1\end{array}$ & $\begin{array}{l}P_{I N T} \text { with } \\
\text { "years as a } \\
\text { smoker" } 1\end{array}$ & $\begin{array}{l}P_{I N T} \text { with } \\
\text { "packs smoked } \\
\text { per day" } 1\end{array}$ & $\begin{array}{l}P_{I N T} \text { with "hours } \\
\text { as a passive } \\
\text { smoker per } \\
\text { week" } 1^{1}\end{array}$ \\
\hline rs7903146 & 10 & 114758349 & $\mathrm{~T}$ & C & TCF7L2 & 0.0236 & 0.337546 & 0.828909 & 0.468614 & 0.731794 & 0.430069 \\
\hline rs231362 & 11 & 2691471 & G & $A$ & KCNQ1 ${ }^{2}$ & 0.0958 & 0.425727 & 0.524149 & 0.459015 & 0.490526 & 0.839291 \\
\hline rs1552224 & 11 & 72433098 & $A$ & C & ARAP1 & 0.0663 & 0.769243 & 0.910252 & 0.66893 & 0.787785 & 0.317376 \\
\hline rs1387153 & 11 & 92673828 & $\mathrm{~T}$ & C & -------- & 0.4563 & 0.384234 & 0.306898 & 0.417016 & 0.198646 & 0.39214 \\
\hline rs10842994 & 12 & 27965150 & C & $\mathrm{T}$ & LOC105369709 & 0.2070 & 0.597631 & 0.827573 & 0.502432 & 0.904211 & 0.766048 \\
\hline rs1153188 & 12 & 55098996 & $A$ & $\mathrm{~T}$ & -------- & 0.0145 & 0.555762 & 0.943046 & 0.524607 & 0.856577 & 0.491495 \\
\hline rs1531343 & 12 & 66174894 & $\mathrm{C}$ & G & RPSAP52 & 0.1091 & 0.484723 & 0.694244 & 0.524799 & 0.863056 & 0.657571 \\
\hline rs7961581 & 12 & 71663102 & C & $\mathrm{T}$ & -------- & 0.2287 & 0.543227 & 0.640958 & 0.216439 & 0.910725 & 0.642499 \\
\hline rs7957197 & 12 & 121460686 & $\mathrm{~T}$ & $A$ & $O A S L$ & 0.0006 & 0.944019 & 0.942204 & 0.942659 & 0.950889 & 0.933128 \\
\hline rs7177055 & 15 & 77832762 & $A$ & G & -------- & 0.3341 & 0.458439 & 0.262651 & 0.502393 & 0.154147 & 0.315986 \\
\hline rs11634397 & 15 & 80432222 & G & $A$ & -------- & 0.0836 & 0.369702 & 0.637139 & 0.599593 & 0.613005 & 0.376819 \\
\hline rs8042680 & 15 & 91521337 & $A$ & $\mathrm{C}$ & PRC1 & 0.0029 & 0.410296 & 0.259713 & 0.229001 & 0.488553 & 0.377059 \\
\hline rs7202877 & 16 & 75247245 & $\mathrm{~T}$ & G & -------- & 0.2050 & 0.53142 & 0.17209 & 0.278141 & 0.284517 & 0.767809 \\
\hline rs12970134 & 18 & 57884750 & $A$ & G & -------- & 0.1738 & 0.274048 & 0.781098 & 0.37251 & 0.629756 & 0.444281 \\
\hline rs10401969 & 19 & 19407718 & C & $\mathrm{T}$ & SUGP1 & 0.0965 & 0.884363 & 0.886034 & 0.825834 & 0.666951 & 0.945 \\
\hline
\end{tabular}

Supplemental Table S20 (The 38 diabetes-associated SNPs identified from European GWASs) SNP-smoking interaction analysis on diabetes status

${ }^{1} P_{I N T}$ is the $p$-value of testing $H_{0}: \beta_{I N T}=0$ vs. $H_{1}: \beta_{I N T} \neq 0$ from the following model:

$$
g[E(Y)]=\beta_{0}+\beta_{S N P} S N P+\beta_{\text {Smoking }} \text { Smoking }+\beta_{I N T} S N P \times \text { Smoking }+\sum_{v=1}^{16} \beta_{C_{v}} \text { Covariate }_{v},
$$

where $Y$ is the diabetes status with a logit link $g[\cdot]$, and Smoking is one of the five smoking measurements. $P_{I N T}<0.05$ was highlighted in 
yellow.

${ }^{2}$ CDC123, KCNQ1, and SLC30A8 were also associated with FG/HbA1c/diabetes according to our discovery cohort (TWB1), as listed in the above Tables S5-S7.

*** SNP rs13266634 (in the SLC30A8 gene) is also among the 6 diabetes-associated SNPs discovered by TWB1, as listed in the above Table S7. 


\begin{tabular}{|c|c|c|c|c|c|c|}
\hline \multirow[b]{2}{*}{$\begin{array}{l}\text { Explanatory variables in } \\
\text { regression model (4) }{ }^{1}\end{array}$} & \multicolumn{2}{|c|}{ Fasting glucose (mg/dL) } & \multicolumn{2}{|c|}{ HbA1c (\%) } & \multicolumn{2}{|c|}{ Diabetes (dichotomous trait) } \\
\hline & $\begin{array}{l}\text { Percent } \\
\text { change }\end{array}$ & $p$-value & $\begin{array}{l}\text { Percent } \\
\text { change }\end{array}$ & $p$-value & Odds ratio & $p$-value \\
\hline $\begin{array}{c}\text { European-derived GRS (EuGRS) } \\
\text { (z-score standardized) }\end{array}$ & 1.17 & $9.1 \mathrm{E}-83$ & 0.79 & $1.5 \mathrm{E}-72$ & 1.23 & $1.3 \mathrm{E}-30$ \\
\hline $\begin{array}{c}\text { Sex } \\
\text { (1: female vs. 0: male) }\end{array}$ & -3.36 & $1.5 \mathrm{E}-123$ & -0.81 & $9.8 \mathrm{E}-15$ & 0.70 & 2.7E-23 \\
\hline $\begin{array}{c}\text { Age } \\
\text { (in years, continuous variable) }\end{array}$ & 0.36 & 0 & 0.29 & 0 & 1.08 & 0 \\
\hline $\begin{array}{c}\text { Body mass index } \\
\text { (in } \mathrm{kg} / \mathrm{m}^{2} \text {, continuous variable) }\end{array}$ & 0.85 & 0 & 0.73 & 0 & 1.18 & 0 \\
\hline $\begin{array}{c}\text { Active smoking status } \\
\text { (1: yes vs. } 0 \text { : no) }\end{array}$ & 0.51 & 0.13 & 0.87 & $4.2 \mathrm{E}-4$ & 1.21 & 0.06 \\
\hline $\begin{array}{c}\text { EuGRS } \times \text { active smoking status } \\
\text { (continuous variable) }\end{array}$ & 0.4836 & 0.0305 & 0.4738 & 0.0036 & 1.0782 & 0.1474 \\
\hline $\begin{array}{l}\text { Drinking status } \\
\text { (1: yes vs. } 0: \text { no) }\end{array}$ & 2.08 & 3.3E-11 & -0.91 & $5.9 \mathrm{E}-5$ & 0.91 & 0.21 \\
\hline $\begin{array}{l}\text { Regular exercise } \\
\text { (1: yes vs. } 0: \text { no) }\end{array}$ & -0.90 & 4.7E-12 & -0.98 & $5.7 \mathrm{E}-25$ & 0.87 & $6.9 \mathrm{E}-6$ \\
\hline $\begin{array}{l}\text { Educational attainment } \\
\text { (a value ranging from } 1 \text { to } 7 \text { ) }\end{array}$ & -0.52 & $1.5 \mathrm{E}-14$ & -0.20 & $3.9 \mathrm{E}-5$ & 0.89 & 7.3E-16 \\
\hline R-square ${ }^{2}$ & & & & & & \\
\hline
\end{tabular}

Supplemental Table S21 EuGRS-smoking interaction analysis based on the TWB2 cohort

1. Natural log transformed fasting glucose (or HbA1c), or diabetes status, was regressed by model (4). European-derived GRS was calculated by EuGRS $=\sum_{j=1}^{38} w_{j} S N P_{j}$, where the weights $\left(w_{j}, j=1, \cdots, 38\right)$ were the effect sizes reported by Said et al. (from eTable 5 of Said MA, Verweij N, van der Harst P. Associations of Combined Genetic and Lifestyle Risks With Incident Cardiovascular Disease and Diabetes in the UK Biobank 
Study. Jama Cardiol 2018;3:693-702). To save space, we here omit the results of the 10 PCs, EuGRS $\times$ Covariates, and Smoking $\times$

\section{Covariates.}

2. For continuous traits, R-square is the proportion of variance in natural log transformed fasting glucose (or HbA1c) that can be explained by the explanatory variables shown in model (4). For the dichotomous trait (diabetes status), we present pseudo R-square, defined as one minus the ratio of the log likelihood with intercepts only, and the log likelihood with all predictors.

\begin{tabular}{|c|c|c|c|c|c|c|}
\hline \multirow[b]{2}{*}{$\begin{array}{l}\text { Smoking measurements in regression model } \\
\text { (4) }\end{array}$} & \multicolumn{2}{|c|}{ Fasting glucose (mg/dL) } & \multicolumn{2}{|c|}{ HbA1c (\%) } & \multicolumn{2}{|c|}{ Diabetes (dichotomous trait) } \\
\hline & $\begin{array}{c}\text { Percent } \\
\text { change (\%) }\end{array}$ & $P_{I N T}$ & $\begin{array}{c}\text { Percent } \\
\text { change (\%) }\end{array}$ & $P_{I N T}$ & Odds ratio & $P_{I N T}$ \\
\hline EuGRS $\times$ Active smoking status & 0.4836 & 0.0305 & 0.4738 & 0.0036 & 1.0782 & 0.1474 \\
\hline EUGRS $\times$ The number of pack-years & 0.0188 & 0.0224 & 0.0135 & 0.0244 & 0.9986 & 0.3611 \\
\hline EuGRS $\times$ Years as a smoker & 0.0182 & 0.0215 & 0.0178 & 0.0020 & 1.0010 & 0.5098 \\
\hline EuGRS $\times$ Packs smoked per day & 0.4685 & 0.0582 & 0.3017 & 0.0939 & 0.9796 & 0.6819 \\
\hline $\begin{array}{c}\text { EuGRS } \times \text { Hours as a passive smoker per } \\
\text { week }\end{array}$ & 0.0096 & 0.5720 & -0.0040 & 0.7463 & 0.9993 & 0.8559 \\
\hline
\end{tabular}

Supplemental Table S22 EuGRS-smoking interaction analysis based on the TWB2 cohort (replacing "active smoking status" with the 4 continuous smoking measurements) 


\begin{tabular}{|c|c|c|c|c|}
\hline & \multicolumn{2}{|c|}{ Fasting glucose (mg/dL) } & \multicolumn{2}{|c|}{ HbA1c (\%) } \\
\hline $\begin{array}{c}\text { Smoking measurements in regression } \\
\text { model (4) }\end{array}$ & $\widehat{\phi}_{I N T}$ & $P_{I N T}$ & $\widehat{\phi}_{I N T}$ & $P_{I N T}$ \\
\hline EuGRS $\times$ Active smoking status & 0.8416 & 0.0055 & 0.0351 & 0.0026 \\
\hline EuGRS $\times$ The number of pack-years & 0.0351 & 0.0017 & 0.0012 & 0.0051 \\
\hline EuGRS $\times$ Years as a smoker & 0.0323 & 0.0027 & 0.0014 & 0.0009 \\
\hline EuGRS $\times$ Packs smoked per day & 0.8505 & 0.0111 & 0.0267 & 0.0383 \\
\hline $\begin{array}{c}\text { EuGRS } \times \text { Hours as a passive smoke } \\
r \text { per week }\end{array}$ & 0.0189 & 0.4143 & $-1.6 \mathrm{E}-6$ & 0.9985 \\
\hline
\end{tabular}

Supplemental Table S23 EuGRS-smoking interaction analysis based on the TWB2 cohort (without natural log transformation on fasting glucose or HbA1c) 


\begin{tabular}{|c|c|c|c|c|c|c|}
\hline \multirow[b]{2}{*}{$\begin{array}{l}\text { Smoking measurements in regression } \\
\text { model (4) }\end{array}$} & \multicolumn{2}{|c|}{ Fasting glucose (mg/dL) } & \multicolumn{2}{|c|}{ HbA1c (\%) } & \multicolumn{2}{|c|}{ Diabetes (dichotomous trait) } \\
\hline & $\begin{array}{l}\text { Percent change } \\
\text { (\%) }\end{array}$ & $P_{I N T}$ & $\begin{array}{l}\text { Percent change } \\
\text { (\%) }\end{array}$ & $P_{I N T}$ & Odds ratio & $P_{I N T}$ \\
\hline EuGRS $\times$ Active smoking status & 0.4780 & 0.0243 & 0.4472 & 0.0038 & 1.0637 & 0.1982 \\
\hline EuGRS $\times$ The number of pack-years & 0.0264 & 0.0008 & 0.0191 & 0.0009 & 0.9991 & 0.5279 \\
\hline EuGRS $\times$ Years as a smoker & 0.0227 & 0.0026 & 0.0210 & 0.0001 & 1.0012 & 0.4091 \\
\hline EuGRS $\times$ Packs smoked per day & 0.6280 & 0.0080 & 0.4363 & 0.0114 & 0.9882 & 0.8005 \\
\hline $\begin{array}{c}\text { EuGRS } \times \text { Hours as a passive smoke } \\
r \text { per week }\end{array}$ & 0.0041 & 0.8054 & -0.0073 & 0.5500 & 0.9984 & 0.6701 \\
\hline
\end{tabular}

Supplemental Table S24 EuGRS-smoking interaction analysis based on the TWB2 cohort (assess the EuGRS-smoking interaction without controlling for the other interaction terms) 


\begin{tabular}{|c|c|c|c|c|c|c|c|}
\hline \multirow[b]{2}{*}{$\begin{array}{l}\text { Smoking measurements in regression } \\
\text { model (4) }\end{array}$} & \multirow[b]{2}{*}{ Sex } & \multicolumn{2}{|c|}{ Fasting glucose (mg/dL) } & \multicolumn{2}{|c|}{ HbA1c (\%) } & \multicolumn{2}{|c|}{ Diabetes (dichotomous trait) } \\
\hline & & $\begin{array}{l}\text { Percent change } \\
\text { (\%) }\end{array}$ & $P_{I N T}$ & $\begin{array}{l}\text { Percent change } \\
\text { (\%) }\end{array}$ & $P_{I N T}$ & Odds ratio & $P_{I N T}$ \\
\hline \multirow[t]{2}{*}{ EuGRS $\times$ Active smoking status } & Males & 0.6478 & 0.0289 & 0.4822 & 0.0275 & 1.0625 & 0.2719 \\
\hline & Females & 0.0326 & 0.9355 & 0.4313 & 0.1389 & 1.0801 & 0.5509 \\
\hline \multirow[t]{2}{*}{ EuGRS $\times$ The number of pack-years } & Males & 0.0267 & 0.0065 & 0.0170 & 0.0185 & 0.9995 & 0.7611 \\
\hline & Females & -0.0449 & 0.0980 & -0.0123 & 0.5314 & 0.9890 & 0.1028 \\
\hline \multirow[t]{2}{*}{ EUGRS $\times$ Years as a smoker } & Males & 0.0224 & 0.0227 & 0.0180 & 0.0127 & 1.0013 & 0.4231 \\
\hline & Females & -0.0052 & 0.7794 & 0.0140 & 0.2951 & 0.9999 & 0.9782 \\
\hline \multirow[t]{2}{*}{ EuGRS $\times$ Packs smoked per day } & Males & 0.7485 & 0.0148 & 0.4098 & 0.0701 & 0.9954 & 0.9286 \\
\hline & Females & -0.7615 & 0.2201 & -0.1275 & 0.7767 & 0.9733 & 0.2943 \\
\hline \multirow{2}{*}{$\begin{array}{c}\text { EuGRS } \times \text { Hours as a passive smoker } \\
\text { per week }\end{array}$} & Males & 0.0338 & 0.3495 & 0.0181 & 0.4968 & 1.0004 & 0.9446 \\
\hline & Females & 0.0027 & 0.8911 & -0.0129 & 0.3560 & 0.9978 & 0.6485 \\
\hline
\end{tabular}

Supplemental Table S25 EuGRS-smoking interaction analysis based on the TWB2 cohort (Results of EuGRS-smoking interactions while stratified by sex) 

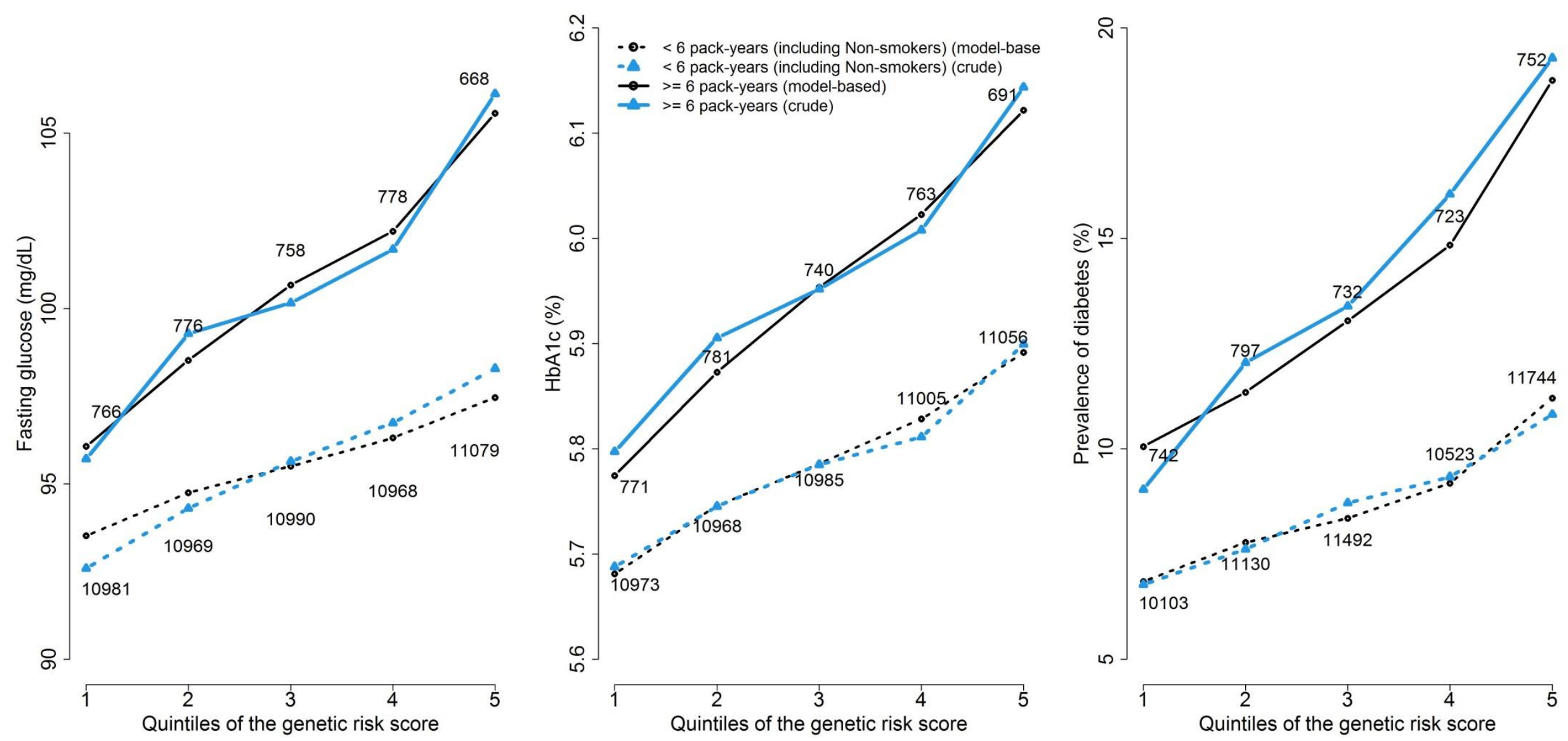

Supplemental Figure S1 Average of FG/HbA1c and the prevalence of diabetes stratified by the number of pack-years and the quintiles of the FG/HbA1c/diabetes genetic risk score

The solid lines are for smokers with >= 6 pack-years, where 6 is the first quartile (Q1) of the number of pack-years in TWB2 smokers (we chose Q1 as the dichotomized point, to prevent a too small sample size in the higher pack-year group). The dotted lines are for non-smokers and smokers with < 6 pack-years. The black lines depict predicted mean FG/HbA1c or predicted prevalence of diabetes based on model (4). Only subjects without any missing in covariates can be predicted. The blue lines mark crude mean FG/HbA1c or crude prevalence of diabetes, without adjusting for any covariates. The number shown around each point represents the sample size of that category. 

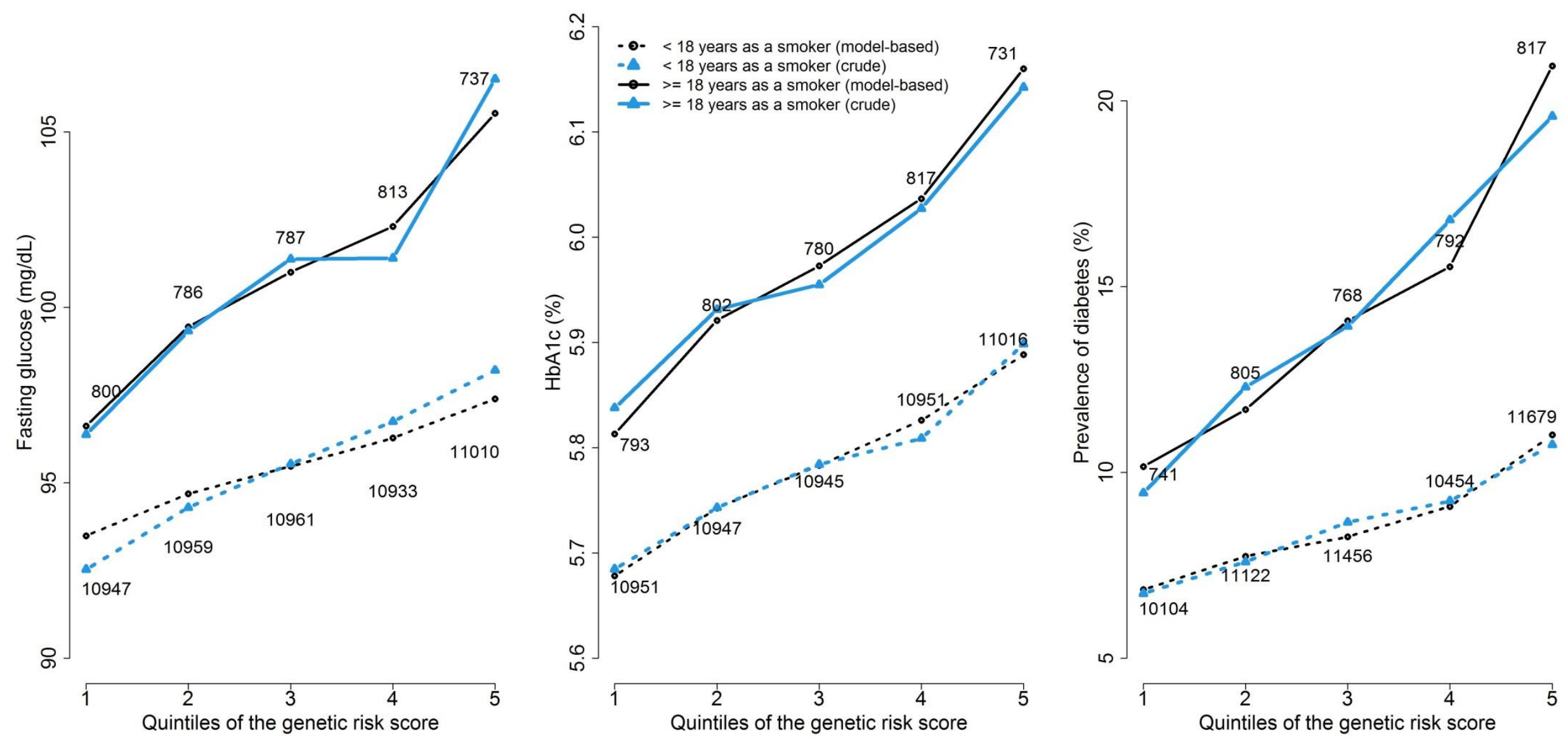

Supplemental Figure S2 Average of FG/HbA1c and the prevalence of diabetes stratified by the number of years as a smoker and the quintiles of the FG/HbA1c/diabetes genetic risk score

The solid lines are for smokers with >= 18 years as a smoker, where 18 is the first quartile (Q1) of the number of years as a smoker in TWB2 smokers (we chose Q1 as the dichotomized point, to prevent a too small sample size in the higher smoking-year group). The dotted lines are for non-smokers and smokers with < 18 years as a smoker. The black lines depict predicted mean FG/HbA1c or predicted prevalence of diabetes based on model (4). Only subjects without any missing in covariates can be predicted. The blue lines mark crude mean FG/HbA1c or crude prevalence of diabetes, without adjusting for any covariates. The number shown around each point represents the sample size of that category. 

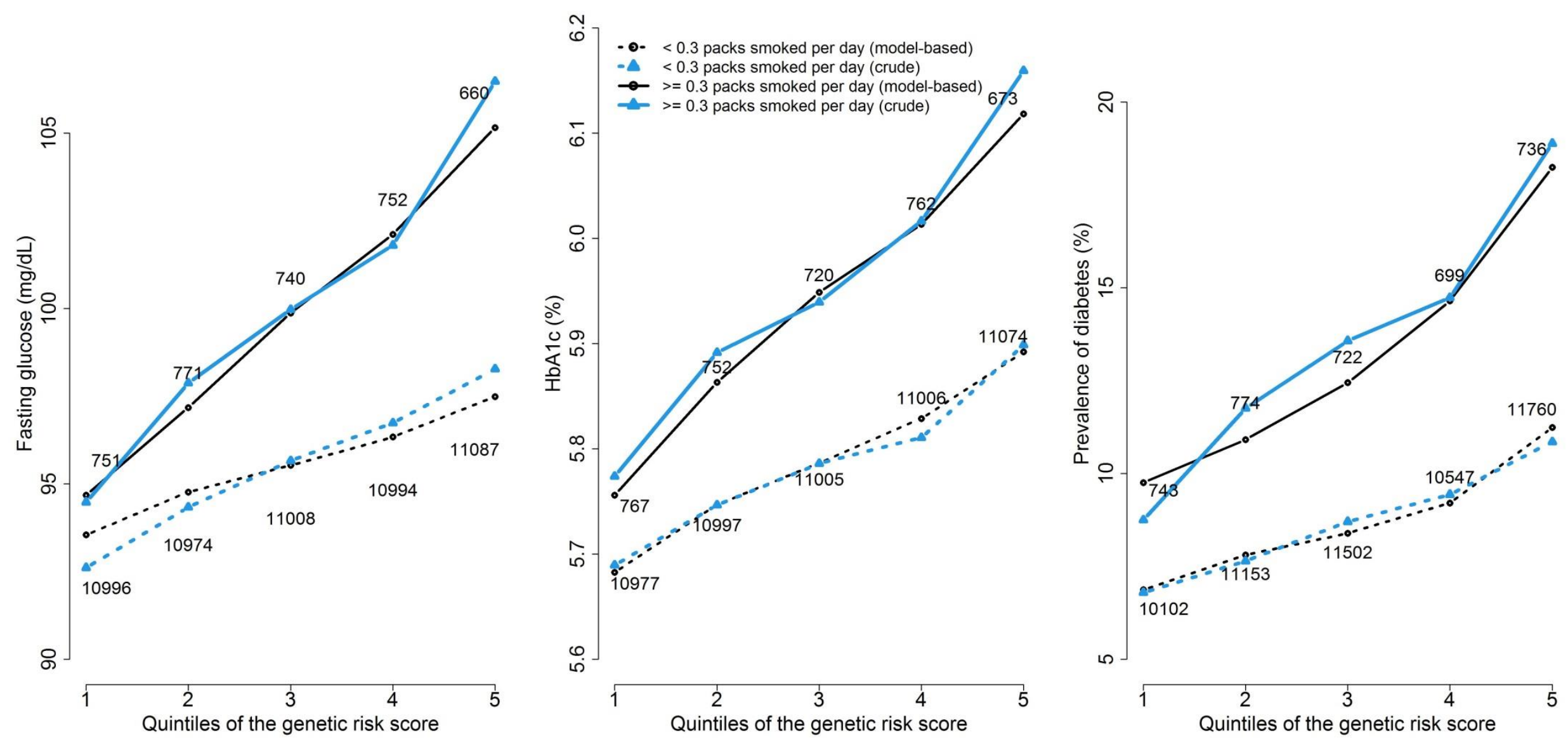

Supplemental Figure S3 Average of FG/HbA1c and the prevalence of diabetes stratified by the number of packs smoked per day and the quintiles of the $\mathrm{FG} / \mathrm{HbA1c}$ /diabetes genetic risk score

The solid lines are for smokers with >= 0.3 packs smoked per day, where 0.3 is the first quartile (Q1) of the number of packs smoked per day in TWB2 smokers (we chose Q1 as the dichotomized point, to prevent a too small sample size in the higher smoking-amount group). The dotted lines are for non-smokers and smokers with $<0.3$ packs smoked per day. The black lines depict predicted mean FG/HbA1c or predicted prevalence of diabetes based on model (4). Only subjects without any missing in covariates can be predicted. The blue lines mark crude mean FG/HbA1c or crude prevalence of diabetes, without adjusting for any covariates. The number shown around each point represents the sample size of that category. 

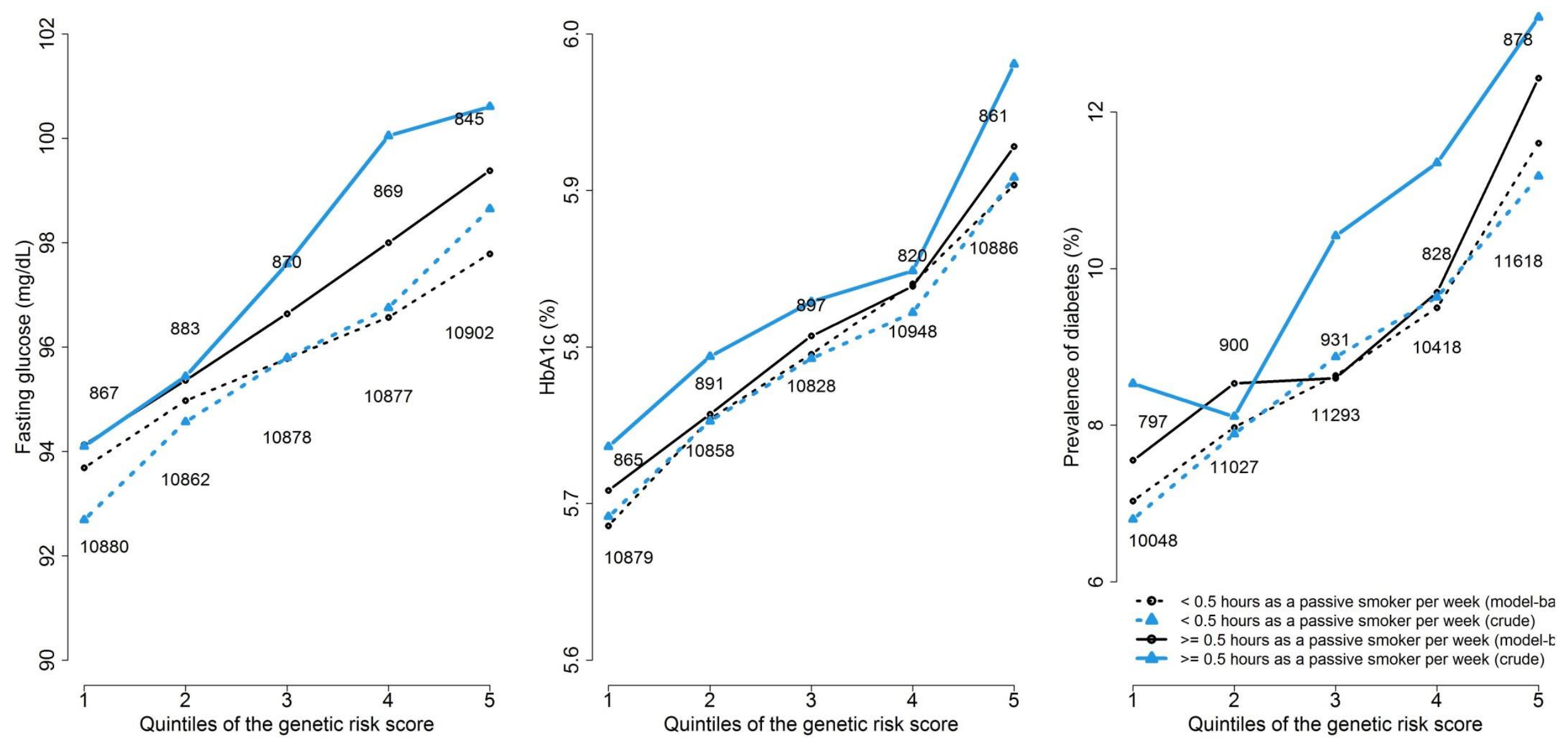

Supplemental Figure S4 Average of FG/HbA1c and the prevalence of diabetes stratified by the number of hours as a passive smoker per week and the quintiles of the $\mathrm{FG} / \mathrm{HbA} 1 \mathrm{c} /$ diabetes genetic risk score

The solid lines are for smokers with >= 0.5 hours as a passive smoker per week, where 0.5 is the first quartile (Q1) of the number of hours as a passive smoker per week in TWB2 passive smokers (we chose Q1 as the dichotomized point, to prevent a too small sample size in the higher passive-smoking-amount group). The dotted lines are for non-passive-smokers and passive smokers with $<0.5$ hours as a passive smoker per week. The black lines depict predicted mean FG/HbA1c or predicted prevalence of diabetes based on model (4). Only subjects without any missing in covariates can be predicted. The blue lines mark crude mean FG/HbA1c or crude prevalence of diabetes, without adjusting for any covariates. The 
number shown around each point represents the sample size of that category. 